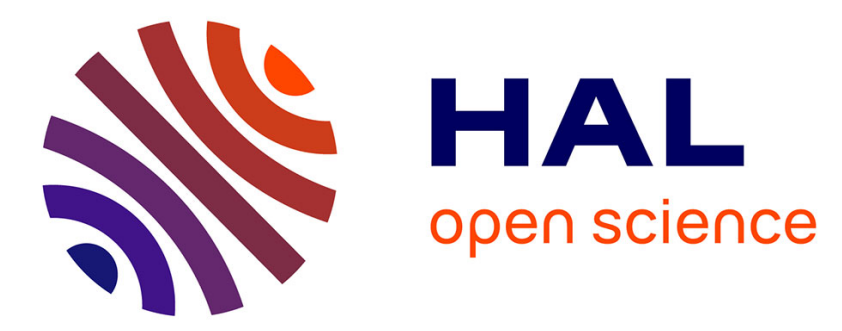

\title{
SpiCee: A Genetic Tool for Subcellular and Cell-Specific Calcium Manipulation
}

Oriol Ros, Sarah Baudet, Yvrick Zagar, Karine Loulier, Fiona Roche, Sandrine Couvet, Alain Aghaie, Melody Atkins, Alice Louail, Christine Petit, et al.

\section{- To cite this version:}

Oriol Ros, Sarah Baudet, Yvrick Zagar, Karine Loulier, Fiona Roche, et al.. SpiCee: A Genetic Tool for Subcellular and Cell-Specific Calcium Manipulation. Cell Reports, 2020, 32 (3), pp.107934. 10.1016/j.celrep.2020.107934 . hal-02992312

\section{HAL Id: hal-02992312 https://hal.science/hal-02992312}

Submitted on 9 Nov 2020

HAL is a multi-disciplinary open access archive for the deposit and dissemination of scientific research documents, whether they are published or not. The documents may come from teaching and research institutions in France or abroad, or from public or private research centers.
L'archive ouverte pluridisciplinaire HAL, est destinée au dépôt et à la diffusion de documents scientifiques de niveau recherche, publiés ou non, émanant des établissements d'enseignement et de recherche français ou étrangers, des laboratoires publics ou privés. 


\section{Cell Reports}

\section{SpiCee: A Genetic Tool for Subcellular and Cell- Specific Calcium Manipulation}

\section{Graphical Abstract}

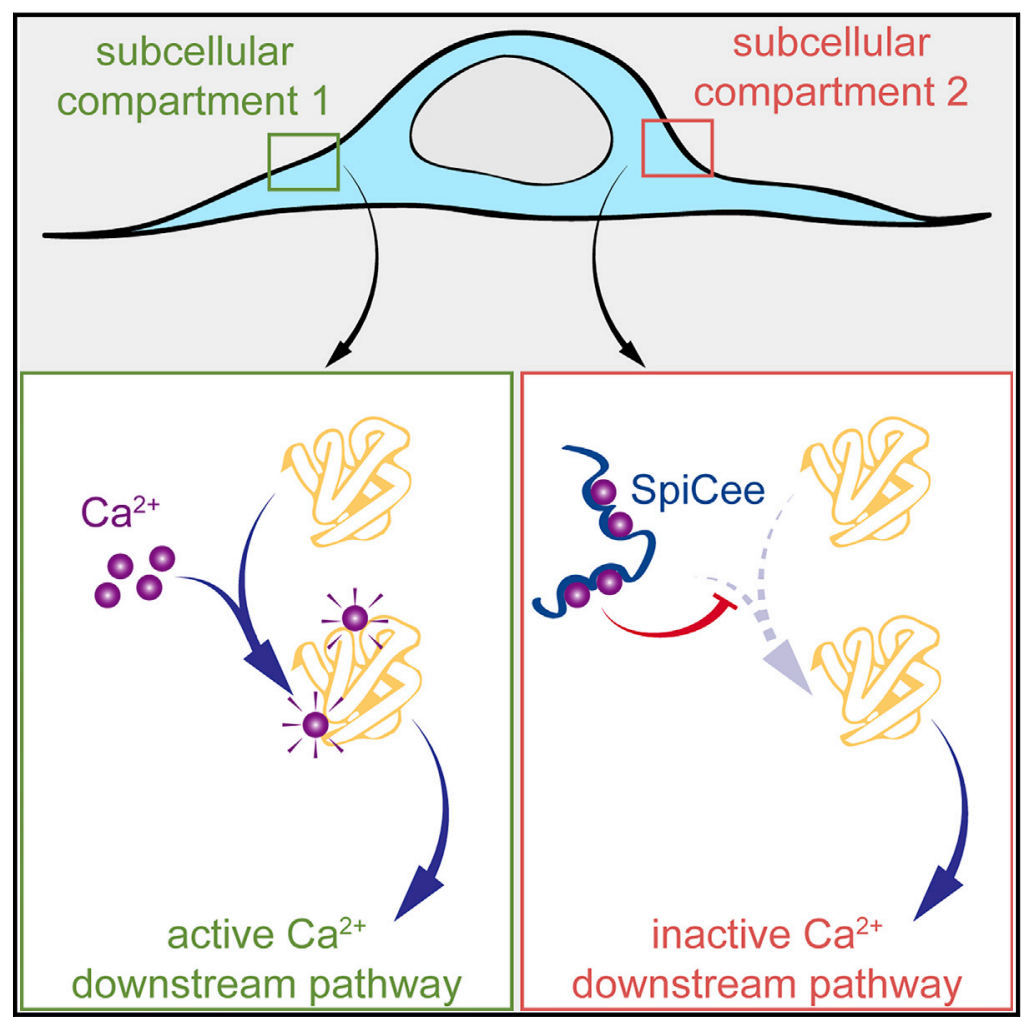

\section{Highlights}

- Development of SpiCee, a genetically encoded calcium scavenger

- SpiCee inhibits calcium-dependent downstream pathways

- SpiCee enables cell-specific manipulation of calciumdependent processes in vivo

- Subcellular targeting confers cell compartment specificity to SpiCee

\section{Authors}

Oriol Ros, Sarah Baudet, Yvrick Zagar, ..., Christine Metin, Yves Mechulam, Xavier Nicol

\section{Correspondence}

xavier.nicol@inserm.fr

\section{In Brief}

Ros et al. develop SpiCee, a genetically encoded calcium chelator that enables the manipulation of this second messenger in single cells with subcellular specificity. SpiCee alters the migration of developing cortical neurons in vivo. Targeting of lipid rafts prevents axonal repulsion, whereas exclusion from this subcellular compartment does not. 


\title{
SpiCee: A Genetic Tool for Subcellular and Cell-Specific Calcium Manipulation
}

\author{
Oriol Ros, ${ }^{1,6}$ Sarah Baudet, ${ }^{1,6}$ Yvrick Zagar, ${ }^{1}$ Karine Loulier, ${ }^{1}$ Fiona Roche, ${ }^{1}$ Sandrine Couvet, ${ }^{1}$ Alain Aghaie, ${ }^{2}$ \\ Melody Atkins, ${ }^{3}$ Alice Louail, ${ }^{1}$ Christine Petit, ${ }^{2,4}$ Christine Metin, ${ }^{3}$ Yves Mechulam, ${ }^{5}$ and Xavier Nicol ${ }^{1,7, *}$ \\ ${ }^{1}$ Sorbonne Université, INSERM, CNRS, Institut de la Vision, 17 rue Moreau, 75012 Paris, France \\ 2INSERM, Sorbonne Université, Institut Pasteur, UMR_S 1120, 75012 Paris, France \\ ${ }^{3}$ INSERM, UMR-S839, Sorbonne Université, Institut du Fer à Moulin, 75005 Paris, France \\ ${ }^{4}$ Collège de France, 75005 Paris, France \\ ${ }^{5}$ Laboratoire de Biochimie, Ecole Polytechnique, CNRS UMR 7654, 91128 Palaiseau, France \\ ${ }^{6}$ These authors contributed equally \\ ${ }^{7}$ Lead Contact \\ *Correspondence: xavier.nicol@inserm.fr \\ https://doi.org/10.1016/j.celrep.2020.107934
}

\section{SUMMARY}

Calcium is a second messenger crucial to a myriad of cellular processes ranging from regulation of metabolism and cell survival to vesicle release and motility. Current strategies to directly manipulate endogenous calcium signals lack cellular and subcellular specificity. We introduce SpiCee, a versatile and genetically encoded chelator combining low- and high-affinity sites for calcium. This scavenger enables altering endogenous calcium signaling and functions in single cells in vitro and in vivo with biochemically controlled subcellular resolution. SpiCee paves the way to investigate local calcium signaling in vivo and directly manipulate this second messenger for therapeutic use.

\section{INTRODUCTION}

Alterations of calcium $\left(\mathrm{Ca}^{2+}\right)$ signals currently rely mostly on pharmacological strategies to prevent $\mathrm{Ca}^{2+}$ influx into the cytoplasm by blocking specific channels or chelate extracellular or intracellular $\mathrm{Ca}^{2+}$ using buffers designed decades ago (Godfraind, 2017; Tsien, 1980). Since $\mathrm{Ca}^{2+}$ is crucial for a wide range of signaling pathways and for the function of many components of the extracellular matrix (Averaimo and Nicol, 2014; Clapham, 2007), these strategies lack cellular specificity leading to ambiguous interpretation of their effects. The rise of optogenetics has provided a range of approaches to increase intracellular $\mathrm{Ca}^{2+}$ including light-sensitive channels (Nagel et al., 2003) and genetically encoded caged $\mathrm{Ca}^{2+}$ (Fukuda et al., 2014). This has been used to control electrical activity in excitable cells and has provided extraordinary knowledge of neuronal interactions as well as opening promising fields of therapeutics (Rajasethupathy et al., 2016; Sengupta et al., 2016; Tønnesen and Kokaia, 2017). These strategies rely on imposing additional $\mathrm{Ca}^{2+}$ elevations but lack the ability to manipulate endogenous $\mathrm{Ca}^{2+}$ levels, reducing the intracellular concentration or targeting release from internal stores. In addition, the use of optogenetics is handicapped in vivo, when light stimulation is technically challenging (e.g., during developmental stages). Reducing endogenous $\mathrm{Ca}^{2+}$ signals with cellular or subcellular specificity is out of the range of the available toolset (including the widely used BAPTA-AM), even though cell-specific manipulation has been successful in reducing the decay time of $\mathrm{Ca}^{2+}$ transients in cardiac myocytes (Wang et al., 2013). Strategies overexpressing a $\mathrm{Ca}^{2+}$ pump to drain this second messenger out of the cytoplasm of astrocytes ( $\mathrm{Yu}$ et al., 2018) or altering inositol 1,4,5-triphosphate $\left(\mathrm{IP}_{3}\right)$ upstream of $\mathrm{Ca}^{2+}$ entry (Uchiyama et al., 2002) have successfully reduced $\mathrm{Ca}^{2+}$ signaling. However, these strategies lack the versatility to target a specific subcellular compartment and to be applicable to $\mathrm{IP}_{3}$-independent $\mathrm{Ca}^{2+}$ modulation, respectively. Genetically encoded $\mathrm{Ca}^{2+}$ indicators also have secondary buffering activity (McMahon and Jackson, 2018), but their designs aim to minimalize this effect and to limit their impact on $\mathrm{Ca}^{2+}$ signaling. The ideal tool with which to manipulate endogenous $\mathrm{Ca}^{2+}$ signaling would (1) reduce free intracellular $\mathrm{Ca}^{2+}$ available to downstream effectors and preclude cellular processes dependent on this second messenger; (2) interact directly with $\mathrm{Ca}^{2+}$ rather than modifying the activity of $\mathrm{Ca}^{2+}$ channels; (3) be genetically encoded to enable selective subcellular localization using fusion to targeting sequences; and (4) enable cellular identification with fluorescent reporters. Here, we introduce SpiCee (sponge inhibiting $\mathrm{Ca}^{2+}$ signaling), a genetically encoded chelator of intracellular $\mathrm{Ca}^{2+}$, which is targetable to subcellular compartments. We show that SpiCee expression is sufficient to alter $\mathrm{Ca}^{2+}$-dependent signaling and cellular processes both in vitro and in vivo and demonstrate the ability of SpiCee to alter $\mathrm{Ca}^{2+}$-dependent cellular behavior with subcellular specificity. 

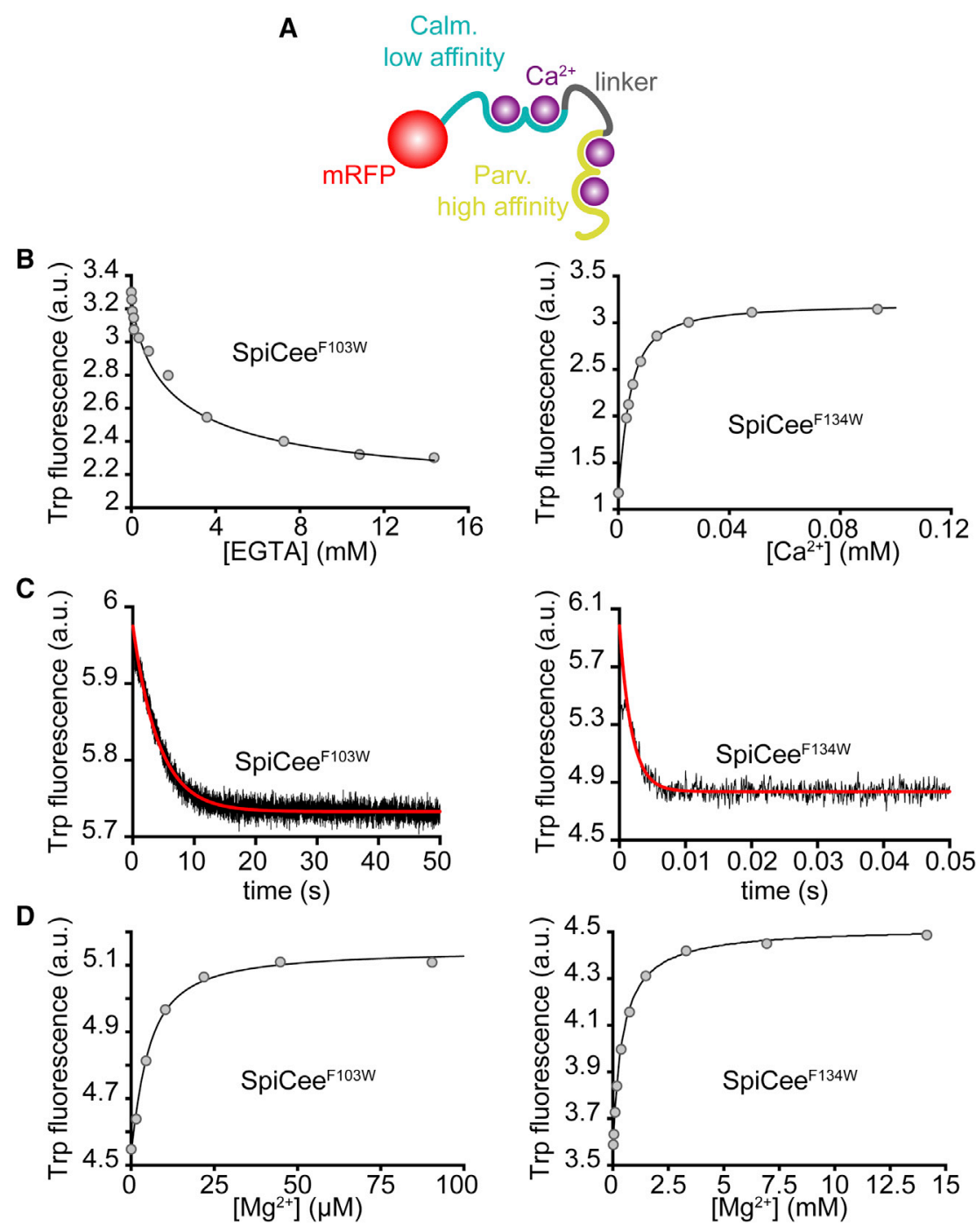

Figure 1. Design and Biochemical Characterization of SpiCee as a $\mathrm{Ca}^{2+}$ Scavenger

(A) SpiCee contains four $\mathrm{Ca}^{2+}$-binding sites, two from calmodulin (Calm.) and two from a high-affinity variant of $\alpha$-parvalbumin (Parv.). The effector domains of calmodulin are absent in SpiCee to avoid the activation of downstream effectors.

(B) SpiCee ${ }^{\mathrm{F} 103 \mathrm{~W}}$ enabled the determination of the dissociation constant $\left(\mathrm{K}_{\mathrm{D}}\right)$ of $\mathrm{Ca}^{2+}$ from the EF-hand adjacent to the inserted tryptophan (residue 103, parvalbumin derived) by competition with EGTA $\left(K_{D}=0.3 \pm 0.1 \mathrm{nM}\right)$, whereas direct $\mathrm{Ca}^{2+}$ titration was used with SpiCee ${ }^{\mathrm{F} 134 \mathrm{~W}}$ to measure the $\mathrm{K}_{\mathrm{D}}$ of the calmodulin-derived $\mathrm{Ca}^{2+}$-binding site in the vicinity of the mutation $\left(\mathrm{K}_{\mathrm{D}}=2.8 \pm 0.9 \mu \mathrm{M}\right)$. Representative titration fits are indicated.

(C) Stopped-flow experiments were conducted to determine the kinetic dissociation constants of $\mathrm{SpiCe}^{\mathrm{F} 103 \mathrm{~W}}$ and SpiCee ${ }^{\mathrm{F} 134 \mathrm{~W}}\left(\mathrm{k}_{\text {off }}=0.24 \pm 0.01 \mathrm{~s}^{-1}\right.$, and $k_{\text {off }}=529 \pm 28 s^{-1}$, respectively). Representative decay curves are shown.

(D) $\mathrm{Mg}^{2+}$ titration was used to measure the dissociation constant of $\mathrm{Mg}^{2+}$ of a parvalbumin- and a calmodulin-derived $\mathrm{Ca}^{2+}$-binding site in the vicinity of the mutations $\left(\mathrm{K}_{\mathrm{D}}=2.6 \pm 0.7 \mu \mathrm{M}\right.$, and $\mathrm{K}_{\mathrm{D}}=0.46 \pm$ $0.14 \mathrm{mM}$, respectively). Representative titration fits are shown. Number of replicates is provided in Table S1. a.u., arbitrary units.

$\mathrm{Ca}^{2+}$-binding properties of the EF-hand adjacent to the mutation, without affecting the affinity of this site (Hutnik et al., 1990; Johnson and Tikunova, 2002; Tang et al., 2003). Each of these mutants enables the evaluation of the $\mathrm{Ca}^{2+}$-binding properties of either the calmodulin or the parvalbumin moiety (SpiCee ${ }^{\mathrm{F} 134 \mathrm{~W}}$ and SpiCee $\mathrm{F}^{\mathrm{F} 103 \mathrm{~W}}$ respectively). Using $\mathrm{Ca}^{2+}$ titration, we found that SpiCee ${ }^{\mathrm{F} 134 \mathrm{~W}}$ has a dissociation constant, $\mathrm{K}_{\mathrm{D}}$, for $\mathrm{Ca}^{2+}$ of $2.8 \mu \mathrm{M} \pm 0.9 \mu \mathrm{M}$, and stopped-flow experiments were per-

\section{RESULTS}

SpiCee Is a Bimodal $\mathrm{Ca}^{2+}$ Chelator

SpiCee was designed as a tetravalent chimeric $\mathrm{Ca}^{2+}$ buffer combining the high-affinity $\mathrm{Ca}^{2+}$-binding domains of a variant of $\alpha$-parvalbumin (Lee et al., 2004) and the lower affinity binding sites of calmodulin (Gifford et al., 2007) (Figure 1A). This design, based on two endogenous proteins with distinct affinities for $\mathrm{Ca}^{2+}$, was chosen to ensure that SpiCee is functional in a wide variety of cellular processes. The effector domains of calmodulin were omitted to avoid gain-of-function effects. SpiCee was fused to the fluorescent mRFP protein for easy identification of SpiCee-expressing cells in fixed- or live-cell experiments (Figure $1 \mathrm{~A})$. The full sequence of SpiCee is provided in the STAR Methods section. To characterize the $\mathrm{Ca}^{2+}$-binding properties of SpiCee, we used the tryptophan fluorescence of two variants of SpiCee (SpiCee ${ }^{\mathrm{F} 134 \mathrm{~W}}$ and SpiCee ${ }^{\mathrm{F} 103 \mathrm{~W}}$ ). The tryptophan fluorescence of similar mutants of the native proteins (calmodulin and parvalbumin) have previously been used to determine the formed to evaluate its dissociation rate, $\mathrm{k}_{\mathrm{off}}$, of $529 \mathrm{~s}^{-1} \pm$ $28 \mathrm{~s}^{-1}$ at $20^{\circ} \mathrm{C}$ (Figures $1 \mathrm{~B}$ and $1 \mathrm{C}$ ). The high affinity of the labeled parvalbumin site $\left(\mathrm{SpiCee}^{\mathrm{F} 103 \mathrm{~W}}\right)$ prevented direct $\mathrm{Ca}^{2+}$ titration, and the $K_{D}$ was measured by competition with EGTA. The $\mathrm{K}_{\mathrm{D}}$ of SpiCee ${ }^{\mathrm{F} 103 \mathrm{~W}}$ is $0.3 \mathrm{nM} \pm 0.1 \mathrm{nM}$, and $\mathrm{k}_{\text {off }}=$ $0.24 \mathrm{~s}^{-1} \pm 0.01 \mathrm{~s}^{-1}$ (Figures $1 \mathrm{~B}$ and $1 \mathrm{C}$ ). $\mathrm{k}_{\mathrm{on}}$ was computed from $\mathrm{K}_{\mathrm{D}}$ and $\mathrm{k}_{\text {off }}\left(k_{\text {on }}=\left(k_{\text {off }} / K_{D}\right)\right) . \mathrm{k}_{\text {on }}=8 \cdot 10^{8} \mathrm{M}^{-1} \mathrm{~s}^{-1} \pm$ $3 \cdot 10^{8} \mathrm{M}^{-1} \mathrm{~s}^{-1}$, and $k_{\mathrm{on}}=1.9 \cdot 10^{8} \mathrm{M}^{-1} \mathrm{~s}^{-1} \pm 0.7 \cdot 10^{7} \mathrm{M}^{-1} \mathrm{~s}^{-1}$ for SpiCee ${ }^{\mathrm{F} 103 \mathrm{~W}}$ (parvalbumin site) and SpiCee ${ }^{\mathrm{F} 134 \mathrm{~W}}$ (calmodulin site), respectively. The $\mathrm{K}_{\mathrm{D}} \mathrm{S}$ for $\mathrm{Mg}^{2+}$ of a parvalbumin-derived and a calmodulin-derived binding site were measured following the tryptophan fluorescence of SpiCee ${ }^{\mathrm{F} 103 \mathrm{~W}}\left(\mathrm{~K}_{\mathrm{D}}=2.6 \mu \mathrm{M} \pm\right.$ $0.7 \mu \mathrm{M})$ and SpiCee ${ }^{\mathrm{F} 134 \mathrm{~W}}\left(\mathrm{~K}_{\mathrm{D}}=0.46 \mathrm{mM} \pm 0.14 \mathrm{mM}\right)$, respectively, highlighting a high selectivity for $\mathrm{Ca}^{2+}$ over $\mathrm{Mg}^{2+}$ (Figure 1D). These features are in the range of the previously described $\mathrm{Ca}^{2+}$-binding properties of the full-length parvalbumin and calmodulin proteins (parvalbumin: $\mathrm{K}_{\mathrm{D}}\left(\mathrm{Ca}^{2+}\right)=4 \mathrm{nM}, \mathrm{K}_{\mathrm{on}}=$ $2.5 \cdot 10^{8} \mathrm{M}^{-1} \mathrm{~s}^{-1}, \mathrm{k}_{\mathrm{off}}=1 \mathrm{~s}^{-1}$, and $\mathrm{K}_{\mathrm{D}}\left(\mathrm{Mg}^{2+}\right)=27 \mu \mathrm{M}$; calmodulin: $\mathrm{K}_{\mathrm{D}}=2.4 \mu \mathrm{M}, \mathrm{k}_{\text {on }}=1 \cdot 10^{8} \mathrm{M}^{-1} \mathrm{~s}^{-1}, \mathrm{k}_{\text {off }}=310 \mathrm{~s}^{-1}$ ) (Johnson and 
A
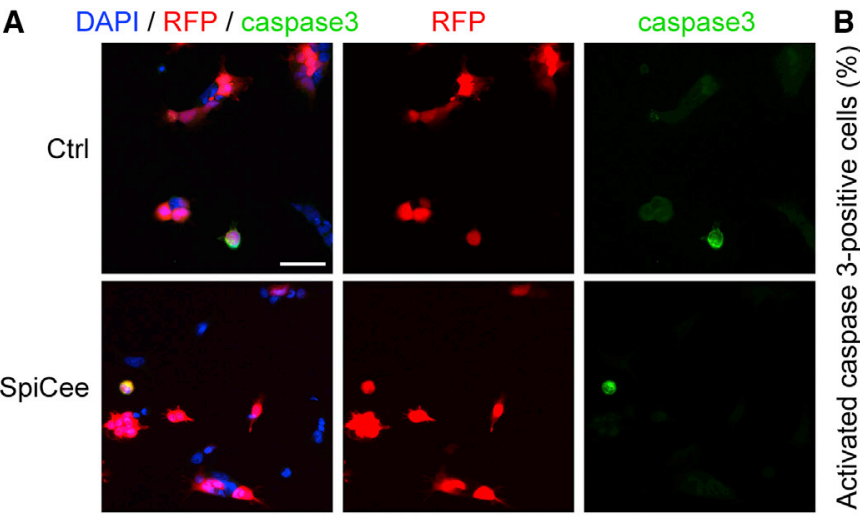

C
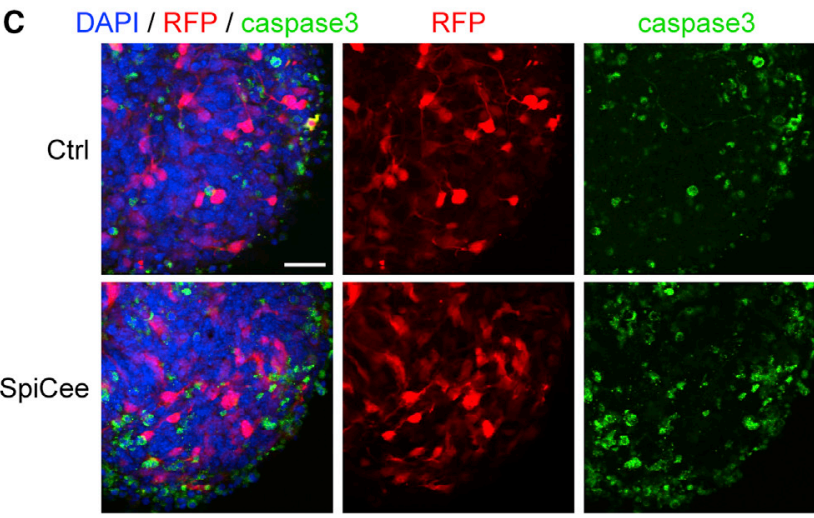

Tikunova, 2002; Lee et al., 2004; Robertson et al., 1981), confirming that SpiCee is a bimodal $\mathrm{Ca}^{2+}$ chelator combining lowand high-affinity sites. The reduced $K_{D}$ of the parvalbumin moiety of SpiCee as compared to the wild-type parvalbumin reflects the use of a high-affinity $\mathrm{Ca}^{2+}$-binding domains of a variant of $\alpha$-parvalbumin (Lee et al., 2004).

\section{SpiCee Is Not Cytotoxic}

SpiCee was expressed in HEK293 cells or in retinal ganglion cells to evaluate its behavior in living cells. Expressing SpiCee did not affect cell survival in either cell type (Figure 2) or the resting $\mathrm{Ca}^{2+}$ concentration in HEK293 cells (Figure S1), suggesting that SpiCee-expressing cells can maintain a resting $\mathrm{Ca}^{2+}$ concentration compatible with their survival, within the previously reported range (Tong et al., 1999). SpiCee-transfected cells were also able to adapt their resting $\mathrm{Ca}^{2+}$ concentration to the amount of $\mathrm{Ca}^{2+}$ in the culture medium (Figure S1). These observations are similar to the reported properties of pharmacological $\mathrm{Ca}^{2+}$ buffers (Pertusa et al., 1999; Tsien, 1980). For instance a few micromolar of BAPTA-AM is able to prevent $\mathrm{Ca}^{2+}$ fluctuations without altering the $\mathrm{Ca}^{2+}$ resting concentration (Collatz et al., 1997).

\section{SpiCee Is a $\mathrm{Ca}^{2+}$ Scavenger in Living Cells}

We investigated SpiCee influence on the dynamic changes in $\mathrm{Ca}^{2+}$ concentration using Twitch2B, an optimized $\mathrm{Ca}^{2+}$ fluorescence resonance energy transfer (FRET) sensor (Thestrup et al., 2014). The FRET:CFP ratio reflects intracellular $\mathrm{Ca}^{2+}$
Figure 2. SpiCee Does Not Affect Cell Survival

(A) HEK293 cells were transfected with either SpiCee or mRFP. Activated caspase-3-positive cells were immunolabeled to evaluate the number of cells undergoing apoptosis. SpiCee-expressing cells are not more prone to enter an apoptotic program than their mRFP-expressing controls.

(B) Fraction of SpiCee- or mRFP-expressing HEK293 cells positive for activated caspase-3.

(C) The survival of retinal ganglion cells, detected by the absence of activated caspase-3, is not affected by SpiCee expression.

(D) Fraction of SpiCee- or mRFP-expressing retinal ganglion cells positive for activated caspase-3.

$\ln (\mathrm{A})$ and $(\mathrm{C})$, scale bars, $25 \mu \mathrm{m}$. In (B) and (D), data are mean \pm SEM with individual data points. Mann-Whitney test. Exact $p$ values and number of replicates are provided in Table S1. See also Figure S1.

concentration and was monitored in Twitch2B-expressing HEK293 cells. The release of intracellular $\mathrm{Ca}^{2+}$ stores induced by a 20 -s pulse of thapsigargin led to a $23 \% \pm 1.8 \%$ increase in the FRET:CFP ratio, which was drastically reduced in cells co-expressing SpiCee $(8.6 \% \pm 0.8 \%$; Figures $3 \mathrm{~A}-3 \mathrm{C}$; Video S1). The reduction of the thapsigargin-induced FRET:CFP ratio reveals that SpiCee competes with other $\mathrm{Ca}^{2+}$-binding proteins, including the biosensor Twitch2B, and prevents them from binding efficiently to $\mathrm{Ca}^{2+}$. To investigate the limits of $\mathrm{Ca}^{2+}$ buffering by SpiCee, cells co-expressing SpiCee and Twitch2B were exposed to longer thapsigargin stimulation (1, 2 , or $5 \mathrm{~min}$ ). In all cases, SpiCee attenuated and delayed the $\mathrm{Ca}^{2+}$ elevation detected by the biosensor (Figures S2A-S2I; Videos S2, $\mathrm{S} 3$, and S4). Extreme intracellular $\mathrm{Ca}^{2+}$ elevations were induced by increasing the extracellular $\mathrm{Ca}^{2+}$ level (from $0.2 \mathrm{mM}$ to $2 \mathrm{mM}$ ) after a prolonged thapsigargin exposure followed by a washout of the drug. SpiCee induces a delay in the $\mathrm{Ca}^{2+}$ elevation detected by Twitch2B (Figures S2J-S2L; Video S5). It does not, however, change the amplitude of $\mathrm{Ca}^{2+}$ elevation of this massive and sustained elevation of $\mathrm{Ca}^{2+}$ concentration, suggesting that SpiCee saturates in this case and confirming that the resting concentration of $\mathrm{Ca}^{2+}$ is not affected in SpiCee-expressing cells (Figures S2J-S2L). To verify that the SpiCee-induced reduction of the $\mathrm{Ca}^{2+}$ elevation requires $\mathrm{Ca}^{2+}$ binding, each SpiCee EF-hand was point-mutated twice (D52A, E63Q, D91A, E102Q, D135A, $\mathrm{E} 146 \mathrm{Q}, \mathrm{D} 171 \mathrm{~A}$, and $\mathrm{E} 182 \mathrm{Q})$ to prevent their ability to bind $\mathrm{Ca}^{2+}$ following previously described mutations of these domains (Piazza et al., 2017; Starovasnik et al., 1992). This variant was termed ${ }^{\text {mut }}$ SpiCee and did not affect the $\mathrm{Ca}^{2+}$ elevation induced by a 1-min pulse of thapsigargin (Figures S2M-S2O).

To assess whether SpiCee reduces $\mathrm{Ca}^{2+}$ elevations induced by endogenous signaling rather than thapsigargin-induced inhibition of $\mathrm{Ca}^{2+}$ transfer from the cytoplasm into the endoplasmic reticulum by the SERCA pump, HEK293 cells were exposed to 
A
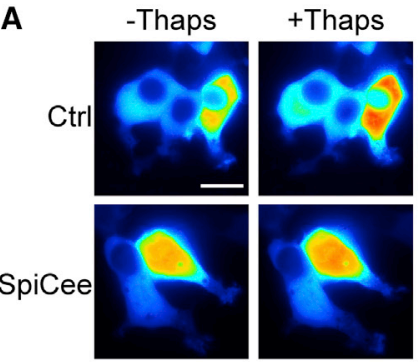

D

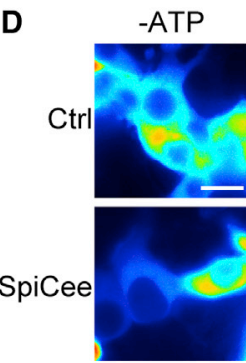

+ATP
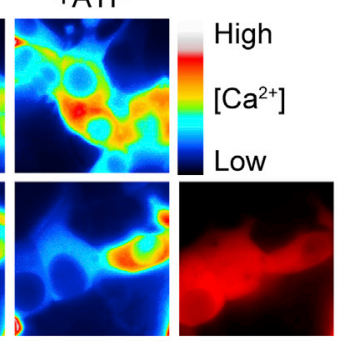

E

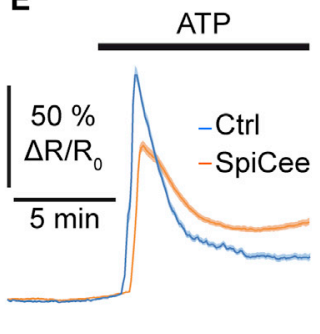

C
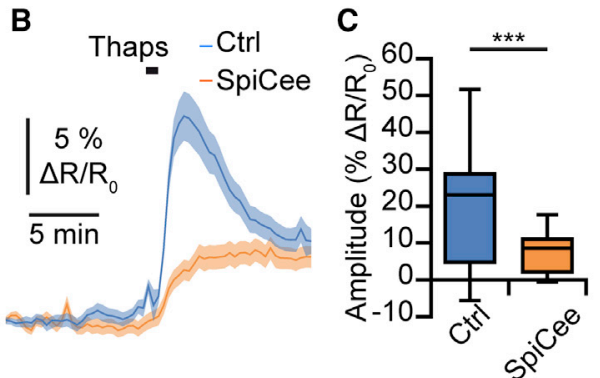

$\mathbf{F}$

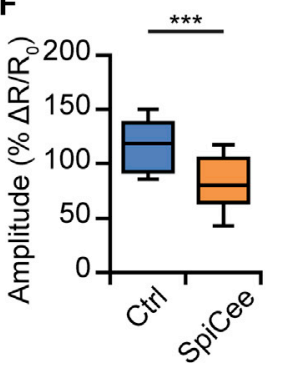

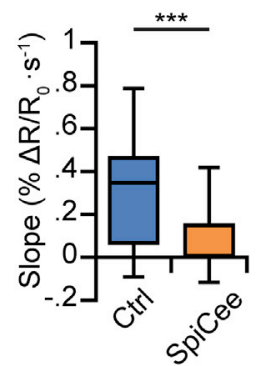

sरे

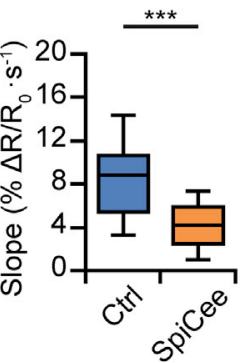

\section{G}

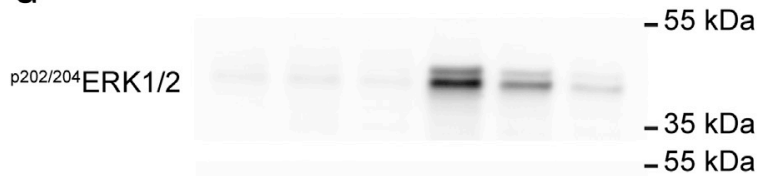

$\mathrm{H}$

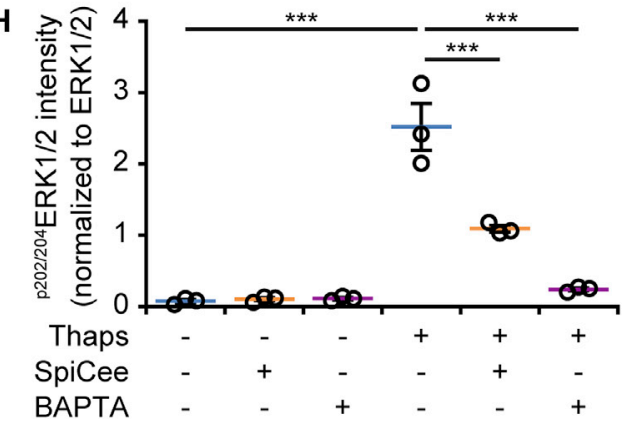

Figure 3. SpiCee Buffers Physiological $\mathrm{Ca}^{2+}$ Transients and Downstream Signaling

(A-C) In vitro experiments show a reduction of the $\mathrm{Ca}^{2+}$ increases induced by thapsigargin. (A and B) 20-s thapsigargin (Thaps.) exposure induces a transient elevation of the $\mathrm{Ca}^{2+}$ concentration in control HEK293 cells, monitored by the FRET:CFP ratio from the FRET biosensor Twitch2B (A, top row, FRET channel; blue trace in B). In contrast, this elevation is drastically reduced in SpiCee-expressing cells (A, bottom row, FRET channel; orange trace in B). (A) The FRET channel intensity is coded from blue (low $\mathrm{Ca}^{2+}$ ) to red (high $\mathrm{Ca}^{2+}$ ); red image indicates SpiCee expression (See also Video S1). (C) Both the amplitude and rate of $\mathrm{Ca}^{2+}$ elevation are reduced by SpiCee expression.

(D-F) ATP superfusion induces a transient elevation of the $\mathrm{Ca}^{2+}$ concentration in control HEK293 cells (D, top row, FRET channel; blue trace in E), monitored by the FRET:CFP ratio from the FRET biosensor Twitch2B. The ATP-induced $\mathrm{Ca}^{2+}$ increase is reduced in SpiCee-expressing cells (D, bottom row, FRET channel; orange trace in E). See also Video S6). (F) Both the amplitude and rate of $\mathrm{Ca}^{2+}$ elevation are decreased by SpiCee expression.

(G) Phosphorylation of ERK1/2 was examined in cells treated with thapsigargin and expressing SpiCee or buffering intracellular Ca ${ }^{2+}$ with BAPTA-AM. Thapsigargin-induced phosphorylation of ERK1/2 was reduced in cells expressing SpiCee, or pre-treated with BAPTA-AM (top), while none of the treatments affected the expression of ERK1/2 (bottom).

(H) Quantification of the western blots shown in $(G)$.

In $(B),(E)$, and $(H)$, data are presented as means \pm SEM. Individual data points are shown in $(H)$. For box-and-whisker plot elements in $(C)$ and $(F)$ : center line, mean; box limits, upper and lower quartiles; whiskers, SD. In (A) and (D), scale bars, $15 \mu \mathrm{m}$. ${ }^{* * *} p \not 0.001$, Mann-Whitney test in (C) and (F) and ANOVA followed by Bonferroni post hoc tests in $(H)$. Exact $p$ values and number of replicates are provided in Table S1. See also Figures S2 and S3.

ATP to increase intracellular $\mathrm{Ca}^{2+}$ concentration through the activation of purinergic receptors (He et al., 2003). SpiCee expression delayed the ATP-induced $\mathrm{Ca}^{2+}$ elevation and reduced its amplitude (Figures 3D-3F; Video S6). To evaluate whether the level of expression of SpiCee is correlated with the extent of $\mathrm{Ca}^{2+}$ scavenging, the amplitude of the ATP-induced $\mathrm{Ca}^{2+}$ elevation for individual cell was plotted against the mRFP intensity as a proxy of SpiCee expression. The FRET:CFP ratio is not correlated with SpiCee expression, suggesting that the scavenging of $\mathrm{Ca}^{2+}$ is achieved from low SpiCee expression (Figure S3A).
These results demonstrate that SpiCee is able to reduce pharmacologically induced $\mathrm{Ca}^{2+}$ elevations and physiological responses over a wide range of intracellular concentrations of this second messenger.

\section{SpiCee Prevents Activation of $\mathrm{Ca}^{2+}$ Downstream Signaling}

We next assessed the ability of SpiCee to preclude signal transduction downstream of $\mathrm{Ca}^{2+}$. Thapsigargin-induced release of $\mathrm{Ca}^{2+}$ from intracellular stores activates the ERK-MAPK 

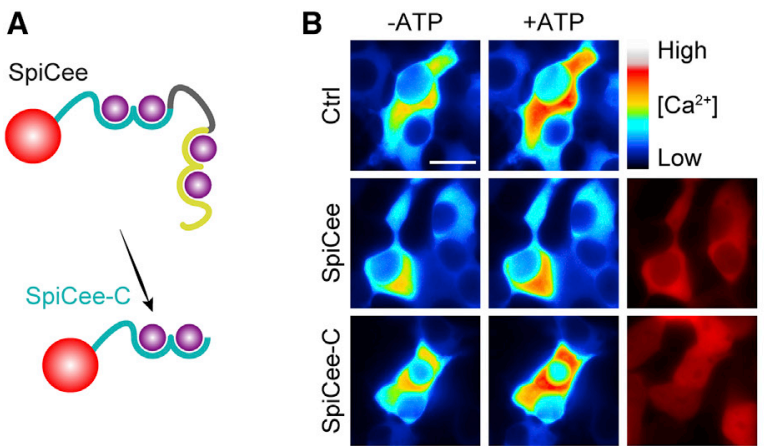

C

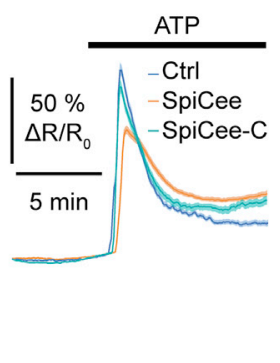

E $\quad F$

F -Thaps

+ Thaps

G

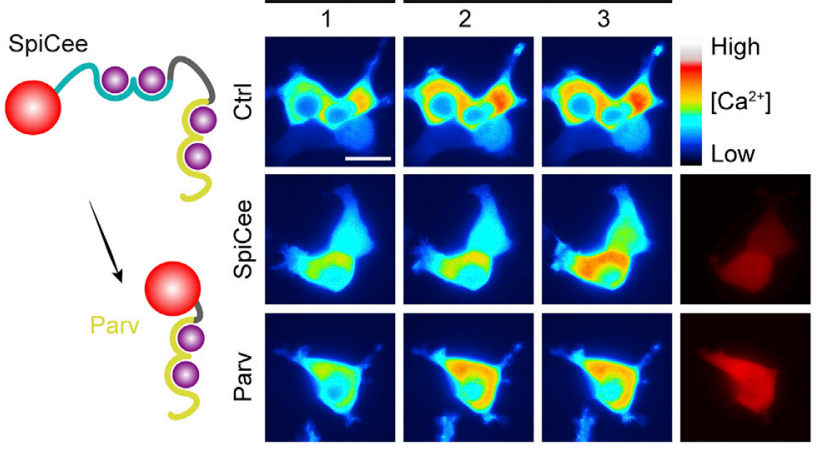

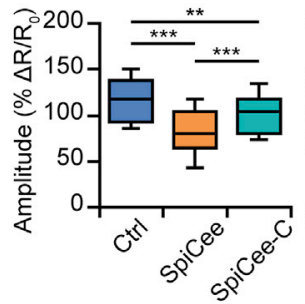

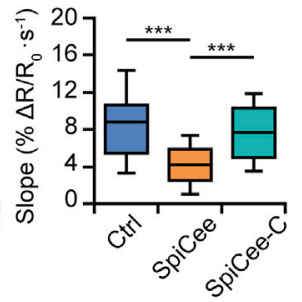

D

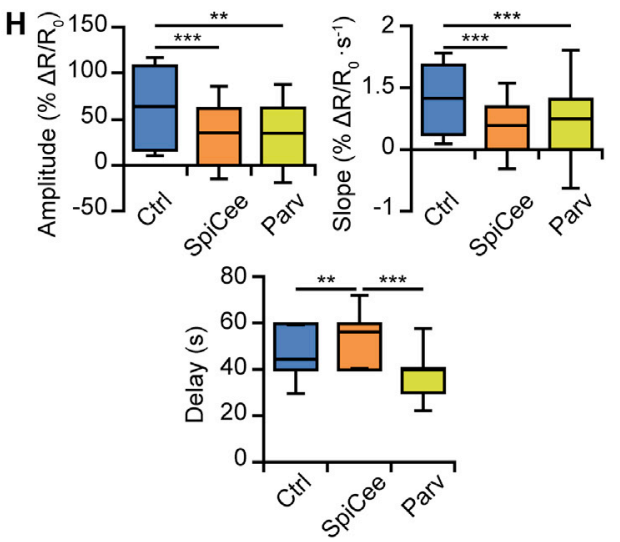

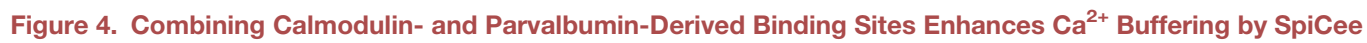

(A) SpiCee-C is a SpiCee variant containing only the $\mathrm{Ca}^{2+}$-binding domain of calmodulin and lacking the parvalbumin-derived $\mathrm{EF}$ hands.

(B and C) mRFP, SpiCee, or SpiCee-C were expressed in HEK293 cells. The FRET:CFP ratio from Twitch2B was monitored before and after superfusion with ATP; red channels in the middle and bottom rows validate SpiCee and SpiCee-C expression, respectively. See also Video S7. ATP induces an elevation in the intracellular $\mathrm{Ca}^{2+}$ concentration in mRFP-expressing cells ( $\mathrm{B}$, top row, FRET channel; blue trace in $\mathrm{C}$ ). SpiCee expression (B, middle row, FRET channel; orange trace in $\mathrm{C})$ is more efficient than SpiCee-C (B, bottom row, FRET channel; cyan trace in $\mathrm{C})$ in reducing this $\mathrm{Ca}^{2+}$ increase.

(D) The reduction of both amplitude and delay of the response to ATP is smaller in SpiCee-C-expressing cells compared to SpiCee-positive cells.

(E) In contrast to SpiCee, parvalbumin (Parv) does not contain EF hands from calmodulin.

( $F$ and G) mRFP, SpiCee, and parvalbumin (Parv) were expressed in HEK293 cells. The FRET:CFP ratio from Twitch2B was monitored before and after superfusion with thapsigargin; red channels in the middle and bottom rows validate SpiCee and parvalbumin expression, respectively. The numbers on top of each column in $(F)$ reflect the time of acquisition for each image and match the position of the numbered time point in (G). Thapsigargin induces an elevation in the intracellular $\mathrm{Ca}^{2+}$ concentration in mRFP-expressing cells ( $F$, top row, FRET channel; blue trace in G). SpiCee expression ( $F$, middle row, FRET channel; orange trace in $\mathrm{G}$ ) delays the onset and reduces the amplitude of this $\mathrm{Ca}^{2+}$ elevation. Parvalbumin also reduces the amplitude but does not affect the onset of the change in $\mathrm{Ca}^{2+}$ concentration ( $\mathrm{F}$, bottom row, FRET channel; green trace in $\mathrm{G}$ ).

In (B) and (F), scale bars, $20 \mu \mathrm{m}$. In (C) and (G), data are means \pm SEM. For box-and-whisker plot elements in (D) and (H): center line, mean; box limits, upper and lower quartiles; whiskers, SD; ${ }^{* *} p<0.01 ;{ }^{* * \star} p<0.001$, Kruskal-Wallis test followed by Mann-Whitney post hoc tests. A subset of the data shown in Figures $3 E$ and $3 F$ is included here for additional comparisons with SpiCee-C. Exact $p$ values and number of replicates are provided in Table S1.

(mitogen-activated protein kinase) pathway (May et al., 2014; Rosado and Sage, 2001). We verified that thapsigargin induces the phosphorylation of ERK1/2, and that this signaling event is abolished by pretreatments with BAPTA-AM (Figures $3 \mathrm{G}$ and $3 \mathrm{H}$ ). Like BAPTA exposure, transfection of SpiCee induced a drastic reduction in the thapsigargin-induced phosphorylation of ERK1/ 2 , demonstrating that SpiCee buffering of $\mathrm{Ca}^{2+}$ prevents the activation of downstream signaling (Figures $3 \mathrm{G}$ and $3 \mathrm{H}$ ). The same approach was used to evaluate whether the $\mathrm{Ca}^{2+}$-binding sites of Twitch2B contribute to the scavenging effect detected using FRET experiments. Co-expressing SpiCee and Twitch2B does not reduce the phosphorylation of ERK1/2 induced by thapsigargin compared to SpiCee expression alone, indicating that Twitch2B does not contribute to the $\mathrm{Ca}^{2+}$ scavenging effect observed using FRET approaches (Figures S3B and S3C).
Combining Low- and High-Affinity Sites Enhances $\mathrm{Ca}^{2+}$ Buffering Ability

To evaluate whether adding two high-affinity $\mathrm{Ca}^{2+}$-binding sites to the pair of lower affinity sites from calmodulin enhances SpiCee performance, a truncated form of SpiCee containing only the calmodulin-derived sites (SpiCee-C) was generated (Figure 4A). SpiCee-C induced a slight reduction in the ATP-induced $\mathrm{Ca}^{2+}$ elevation (Figures 4B-4D; Video S7). The reduction of the $\mathrm{Ca}^{2+}$ signal amplitude was enhanced, and the $\mathrm{Ca}^{2+}$ elevation was delayed when cells express SpiCee instead of SpiCee-C, confirming the superior performance of the 4-binding-site variant of SpiCee over calmodulin-derived $\mathrm{Ca}^{2+}$ buffers like SpiCee-C. SpiCee-C might be adapted for slight attenuation of $\mathrm{Ca}^{2+}$ elevations. 
A DAPI/RFP

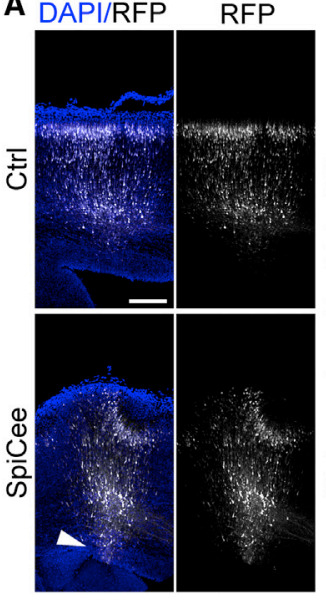

B DAPI/RFP
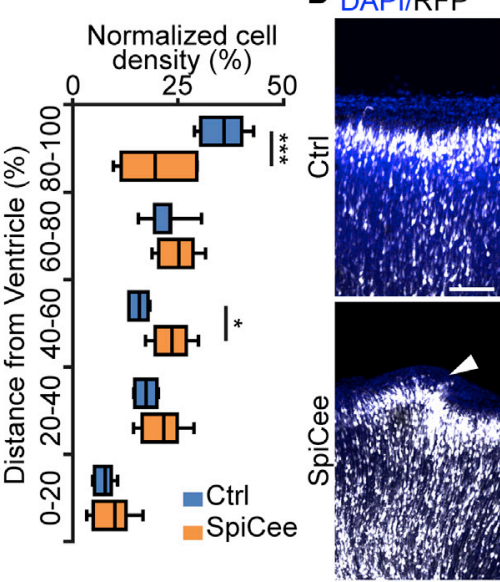
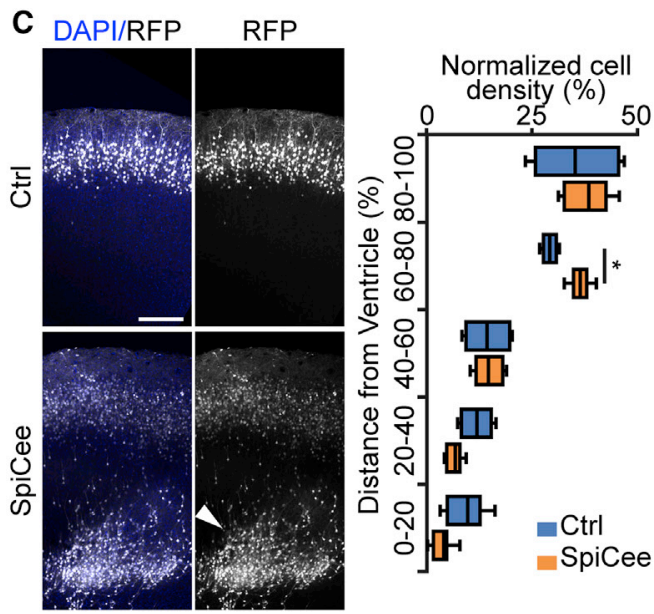

D DAPI/RFP

Figure 5. SpiCee Alters Neuronal Migration In Vivo during Cortical Development

( $A$ and B) Control cortical neurons electroporated at E14.5 form a packed layer close to the marginal zone at E18.5. SpiCee expression interferes with the migration of cortical neurons, as (A) young neurons are scattered across the entire width of the developing cortex (arrowhead), and (B) heterotopic neurons are found at the surface of the cortex (arrowhead).

(C and D) In P10 pups, (C) SpiCee-electroporated neurons spread out more widely in the cortical parenchyma than controls, and (D) heterotopias induced by SpiCee are maintained (arrowhead), indicating that altering $\mathrm{Ca}^{2+}$ signaling impairs cortical neuron migration.

Box-and-whisker plot elements: center line, mean; box limits, upper and lower quartiles; whiskers, SD. Scale bars, $250 \mu \mathrm{m}$ in (A), $100 \mu \mathrm{m}$ in (B), $200 \mu \mathrm{m}$ in (C), and $500 \mu \mathrm{m}$ in (D). ${ }^{*} p \not 0.05 ;{ }^{* \star *} p \not 0.001$; two-way ANOVA and Bonferroni post hoc tests. Exact $p$ values and number of replicates are provided in Table S1. In (C) and (D), images have been automatically stitched to produce the illustrations.

To test whether including the low-affinity binding sites from calmodulin provides an improvement of $\mathrm{Ca}^{2+}$ scavenging by SpiCee over high-affinity buffers like parvalbumin, the thapsigargin-induced $\mathrm{Ca}^{2+}$ elevation was evaluated in SpiCee- and parvalbumin-expressing cells. SpiCee and parvalbumin similarly reduce the amplitude of the Twitch2B FRET:CFP ratio detected after a 1-min thapsigargin pulse. SpiCee also delays the onset of the $\mathrm{Ca}^{2+}$ elevation (Figure $4 \mathrm{E}-4 \mathrm{H}$ ). This delayed $\mathrm{Ca}^{2+}$ elevation was not observed in parvalbumin-expressing cells (Figure 4E$4 \mathrm{H}$ ), suggesting that SpiCee provides enhanced buffering of brief $\mathrm{Ca}^{2+}$ changes.

\section{SpiCee Alters Neuronal Migration In Vivo}

To assess SpiCee capability to interfere with $\mathrm{Ca}^{2+}$-dependent physiological processes in vivo, SpiCee was expressed in newly generated cortical neurons using in utero electroporation of mouse brains at embryonic day 14.5 (E14.5). The migration of early born cortical neurons relies on $\mathrm{Ca}^{2+}$ signaling (Bando et al., 2016; Uhlén et al., 2015). The positions of migrating electroporated neurons from the electroporation site were assessed at E18.5 and post-natal day 10 (P10). At both ages, no obvious morphological defects were detected in SpiCee-expressing neurons. By E18.5, the majority of control GFP and mRFP coelectroporated neurons have reached the cortical plate and formed a dense layer near the marginal zone (Figures $5 \mathrm{~A}$ and 5B). In contrast, many neurons co-expressing GFP and SpiCee exhibited reduced migration and were found throughout the cortical plate, including the intermediate zone (Figure 5A). Several electroporated neurons also failed to stall at the cortical plate, overshooting toward the marginal zone and causing heterotopias in 7 out of 9 animals; misplaced neurons were found in only 2 out of 10 mRFP-electroporated embryos (Figure 5B). At
P10, SpiCee-expressing neurons covered a thicker lamina of the cortex than mRFP-electroporated controls (Figure 5C). Furthermore, the heterotopias detected during embryonic development were maintained at post-natal stages in nearly all SpiCee-expressing animals (10 out of 12), whereas they were found in a single mRFP-electroporated pup (5 electroporated animals; Figure $5 \mathrm{D}$ ). Thus, $\mathrm{Ca}^{2+}$ buffering by SpiCee is sufficient to alter neuronal migration in vivo.

SpiCee Enables Subcellular Manipulation of $\mathrm{Ca}^{2+}$ $\mathrm{Ca}^{2+}$ signals are often restricted to subcellular compartments (Cooper, 2015; Filadi and Pozzan, 2015). Since genetic encoding confers the ability to restrict the expression of the constructs to a specific organelle, we assessed the functionality of SpiCee in this scenario. The ability to restrict SpiCee expression to subcellular compartments was tested using the fusion with targeting sequences or full-length proteins. In developing cortical interneurons, SpiCee is found in the cytoplasm but excluded from the primary cilium. The fusion with transmembrane $5-\mathrm{HT} 6$ receptor leads to the exclusive localization of SpiCee in the primary cilium (Figure S4A). The $\mathrm{Ca}^{2+}$ pump hPMCA (human plasma membrane $\mathrm{Ca}^{2+}$ pump), recently described as a tool to prevent $\mathrm{Ca}^{2+}$ elevation in astrocytes (Yu et al., 2018), was fused to the 5-HT6 receptor to evaluate its ability to be restricted to a membrane compartment. Like SpiCee, 5-HT6-hPMCA was found in the primary cilium. The ability to control the intracellular localization of SpiCee and hPMCA was evaluated in HEK293 cells using a nuclear localization sequence (NLS) and a nuclear export sequence (NES). When not targeted, SpiCee was found in the cytoplasm and in the nucleus with variability in the nuclear level of expression. In contrast, SpiCee-NLS and SpiCee-NES were exclusively found in the nucleus and cytoplasm, respectively, 
A
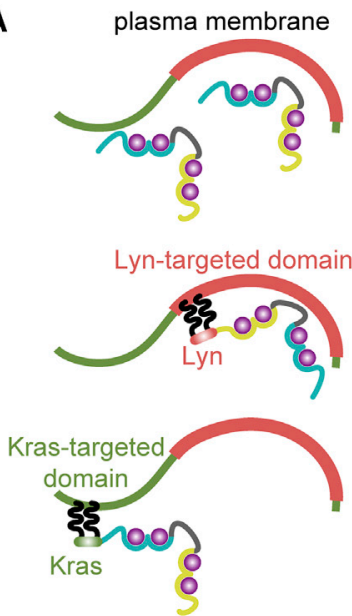

B

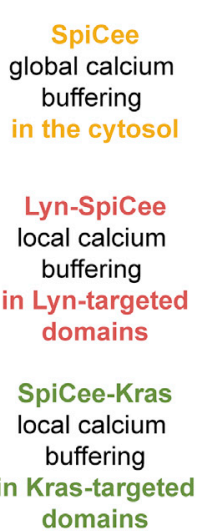

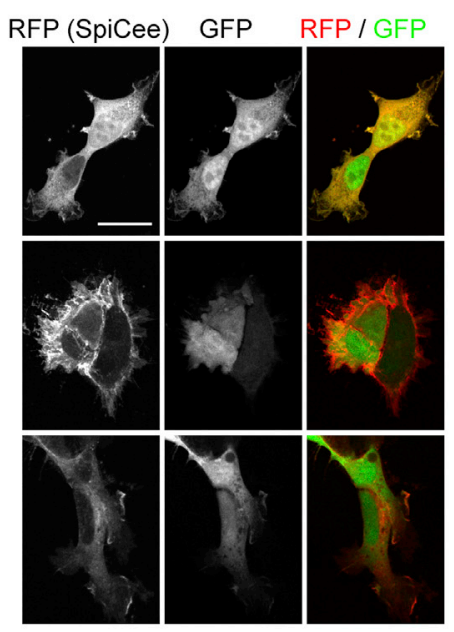

$\mathbf{F}$

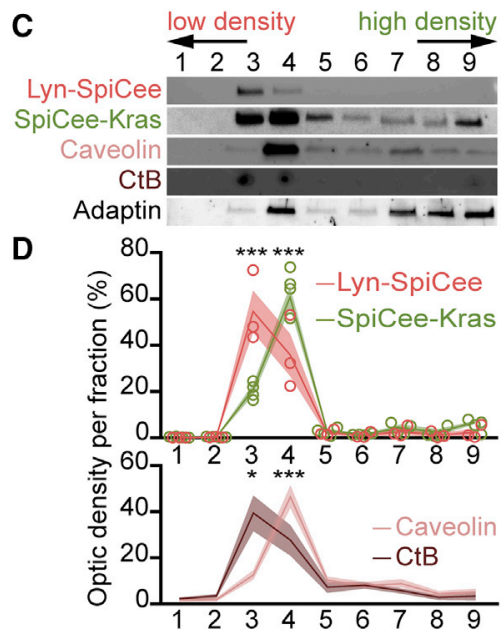

E
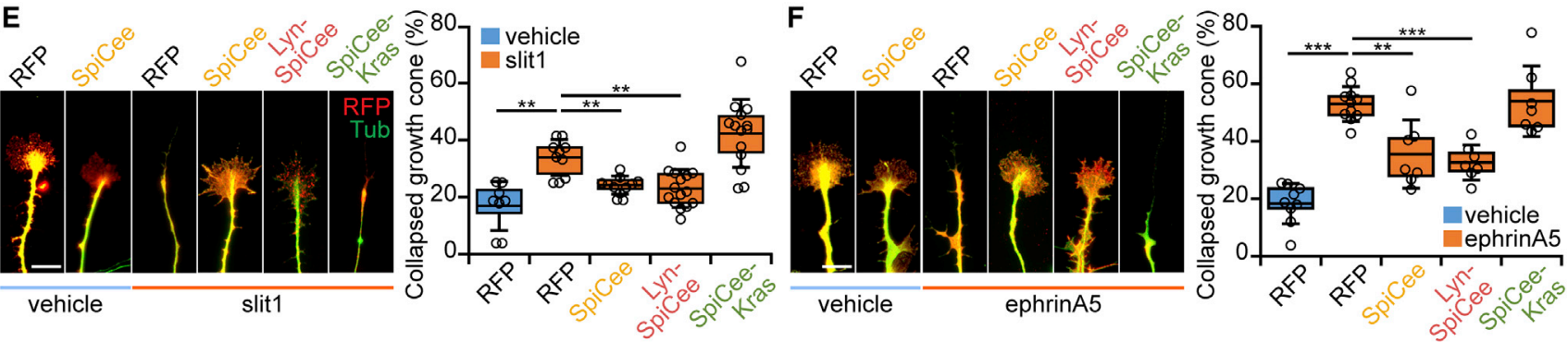

Figure 6. Subcellular Restriction of $\mathrm{Ca}^{2+}$ Manipulation Using SpiCee

SpiCee was either used to globally alter $\mathrm{Ca}^{2+}$ signaling when not targeted to any cellular compartment or to target specific compartments.

(A) Lyn-SpiCee and SpiCee-Kras aim at targeting distinct compartments of the plasma membrane.

(B) SpiCee is detected in the cytoplasm (top row) whether both Lyn-SpiCee and SpiCee-Kras are found at the plasma membrane (middle and bottom rows). (C and D) Plasma membrane fractionation highlighted distinct subcellular localization of Lyn-SpiCee and SpiCee-Kras. Lyn-SpiCee is highly enriched in fraction 3 , like CtB. In contrast, the localization of SpiCee-Kras is shifted to fraction 4, where a distinct membrane marker, Caveolin, is enriched. Surprisingly, it is not restricted to the non-raft fraction of the plasma membrane labeled by Adaptin.

(E and F) Slit1 (E) and (F) ephrinA5 induce growth cone collapse in control axons. SpiCee and Lyn- SpiCee prevent growth cones to collapse. In contrast, SpiCeeKras does not affect axonal response to slit1 and ephrinA5.

In (C), data are means \pm SEM. For box-and-whisker plot elements in (E) and (F): center line, mean; box limits, upper and lower quartiles; whiskers, SD. Scale bars,

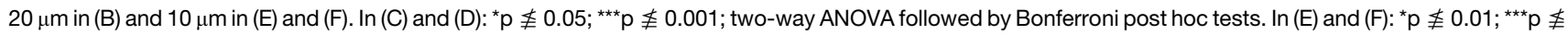
0.001 ; Kruskal-Wallis test followed by Mann-Whitney post hoc tests. Exact $\mathrm{p}$ values and number of replicates are provided in Table $\mathrm{S} 1$. Individual data points missing in Figure 6D are shown in Figure S4 (CtB, for which n $\$$ 10). See also Figures S4, S5, and S6.

demonstrating that its intracellular localization can be controlled (Figure S4B). In the absence of targeting sequences, hPMCA was found in the plasma membrane. The attempts to restrict it to or exclude it from the nucleus using NLS and NES failed, leading to the trapping of hPMCA in a cellular organelle resembling the endoplasmic reticulum (Figure S4B).

In order to directly assess the ability of SpiCee to alter local $\mathrm{Ca}^{2+}$ signaling, a tandem of palmitoylation-myristoylation motifs from Lyn Kinase (Lyn-SpiCee) or the CaaX-polylysine motif derived from K-Ras (SpiCee-Kras) were fused to the $\mathrm{N}$ terminus or the $\mathrm{C}$ terminus of SpiCee, respectively, to target SpiCee to distinct plasma membrane compartments (Figure 6A). Lyn and Kras targeting sequences have previously been used for spatial restriction of overexpressed proteins in lipid rafts and in the nonraft fraction of the plasma membrane, respectively (Averaimo et al., 2016; Depry et al., 2011; Ros et al., 2019; Zacharias et al., 2002). Both Lyn-SpiCee and SpiCee-Kras were targeted to the plasma membrane (Figure $6 \mathrm{~B}$ ). Using sucrose gradient membrane fractionation, Lyn-SpiCee distribution peaked in fraction 3, whereas SpiCee-Kras was highly enriched in fraction 4, demonstrating that each variant of SpiCee is targeted to distinct membrane compartments. Their specific distribution matches the profile of different membrane markers, the $\beta$ subunit of cholera toxin (CtB) and Caveolin respectively, although the biochemical purification used did not localize SpiCee-Kras in the non-raft fraction of the plasma membrane containing Adaptin, a protein excluded from lipid rafts (Figures 6C and 6D; Figure S5).

Using Lyn-SpiCee and SpiCee-Kras, we investigated the potential of SpiCee to manipulate $\mathrm{Ca}^{2+}$-dependent cellular processes specific to subcellular compartments. To this aim, we analyzed the response of retinal ganglion cell axons to the repellent guidance molecules slit1 and ephrinA5, known to require the modulation of $\mathrm{Ca}^{2+}$ concentration (Guan et al., 2007; Nicol et al., 2007) and subcellular-compartment-specific signaling in lipid rafts (Averaimo et al., 2016; Guirland et al., 2004; Nakai and 
Kamiguchi, 2002). In control conditions, including untransfected axons and mRFP-electroporated axons, slit1 and ephrinA5 induced the collapse of non-electroporated or mRFP-expressing growth cones, characterized by the depolymerization of lamellipodial actin and drastic reduction of the growth cone area (Figures $6 \mathrm{E}$ and 6F; Figure S6). SpiCee expression in the cytosol abolished slit1- and ephrinA5-induced growth cone collapse, confirming the requirement of $\mathrm{Ca}^{2+}$ signaling (Figures $6 \mathrm{E}$ and $6 \mathrm{~F}$; Figure S6). Similarly, slit1 or ephrinA5 did not induce the collapse of Lyn-SpiCee-expressing axons (Figures 6E and 6F; Figure S6). In contrast, SpiCee-Kras-expressing axons were indistinguishable from control axons exposed to slit1 or ephrinA5 (Figures 6E and 6F; Figure S6). The ability of SpiCee-Kras to prevent $\mathrm{Ca}^{2+}$ variations was tested using a modified FRET sensor using the same targeting sequence, Twitch2B-Kras. The FRET:CFP ratio of Twitch2B-Kras increases following a 1-minute pulse of thapsigargin. SpiCee expression abolished the transient increase in $\mathrm{Ca}^{2+}$ induced by thapsigargin (Figures S5E-S5G), demonstrating that the scavenging properties of SpiCee are conserved after the fusion to the targeting sequence. These findings demonstrate that the collapse-inducing signaling cascades generated by slit 1 and ephrin $\mathrm{A} 5$ require compartmentalized $\mathrm{Ca}^{2+}$ signaling in distinct domains of the plasma membrane and that targeted versions of SpiCee achieve spatially restricted manipulation of $\mathrm{Ca}^{2+}$-dependent signaling cascades in subcellular compartments.

\section{DISCUSSION}

The present work introduces SpiCee, a molecular scavenger of $\mathrm{Ca}^{2+}$. We show that SpiCee is efficient in buffering $\mathrm{Ca}^{2+}$ transients and downstream signaling, while it does not interfere with the cellular ability to regulate the resting $\mathrm{Ca}^{2+}$ concentration. This resembles the effect of concentrations of BAPTA that prevent the fluctuation of intracellular $\mathrm{Ca}^{2+}$ without altering the resting concentration (Collatz et al., 1997). Since SpiCee can be expressed in a subset of cells, the use of this $\mathrm{Ca}^{2+}$ buffer enables to investigate the role of $\mathrm{Ca}^{2+}$ in neurodevelopmental processes such as neuronal migration in a cell-specific manner. Furthermore, by including the $\mathrm{Ca}^{2+}$-binding domains of endogenous proteins, we have produced a specific and non-cytotoxic buffer that enables chronic manipulation of selected cell types. The possibility of targeting subcellular compartments further increases the ability of SpiCee to affect a subset of $\mathrm{Ca}^{2+}$-dependent downstream pathways with high specificity.

Second messengers are key in the regulation of cellular responses to the environment and of their intrinsic behavior. Being able to manipulate the concentration of these signaling molecules in a precise and predictable fashion is crucial for the understanding of their downstream signaling cascades and cellular responses. Second messengers are located at the convergence of multiple signaling cascades. A simple view of these signaling molecules diffusing freely in the cytosol conflicts with the specific activation of each of their downstream effectors. The spatial confinement of second messengers emerged as a likely regulator of cellular responses (Augustine et al., 2003; Averaimo and Nicol, 2014; Averaimo et al., 2016).
Much effort has been devoted to the development of tools that allow the subcellular manipulation of cyclic nucleotides through genetically encoded tools (Averaimo et al., 2016; Lefkimmiatis et al., 2009; Ros et al., 2019) and $\mathrm{Ca}^{2+}$ using pharmacological agents targeting specific channels. To date, refined ways to block $\mathrm{Ca}^{2+}$ signals in a cell-dependent manner are restricted to the use of genetically encoded $\mathrm{Ca}^{2+}$ pumps (Yu et al., 2018). SpiCee overcomes the issues of current $\mathrm{Ca}^{2+}$-manipulating methods by enabling cellular selectivity when combined with cell-type-specific promoters and targeting of a subcellular localization of choice when fused to known targeting sequences. It offers the possibility to investigate $\mathrm{Ca}^{2+}$ signals to a previously unachieved degree. SpiCee also offers the opportunity to study the epistatic relationship between second messengers in fields like axon guidance, where guidance molecules activating apparently the same signaling cascades are able to exert contradictive actions in developing axons (Akiyama et al., 2016; Nishiyama et al., 2003; Shelly et al., 2010). In brief, SpiCee offers the possibility to study the influence of $\mathrm{Ca}^{2+}$ signaling on the development and function of neuronal networks.

The development of SpiCee-based tools will further expand the possibilities of manipulating $\mathrm{Ca}^{2+}$ with spatial and temporal control. Floxed transgenic rodent strains would allow precise cell-type- and developmental-stage-specific silencing of $\mathrm{Ca}^{2+}$ signaling cascades while facilitating in vivo research by obviating surgical interventions. The temporal control of SpiCee expression could be achieved by genetically encoded switches such as the Tet-ON and Tet-OFF systems. SpiCee might be similarly imported to other animal models such as fly or zebrafish, allowing for the same refined functionality when coupled to upstream activation sequences (UASs) or heat shock elements. When combined with targeting sequences or full-length protein to control its subcellular localization (Kunze and Berger, 2015; Markwardt et al., 2011) and activating or deactivating strategies, SpiCee will improve our understanding of the features of $\mathrm{Ca}^{2+}$ signals that enable the specific control of its downstream effectors.

SpiCee-derived $\mathrm{Ca}^{2+}$ manipulation with subcellular specificity might enable the use of $\mathrm{Ca}^{2+}$ as a therapeutic target with a reduced risk of side effects. However, subcellular $\mathrm{Ca}^{2+}$ manipulation might not be sufficient to achieve full specificity for a single signaling pathway due to the extremely wide range of $\mathrm{Ca}^{2+}$-dependent processes. Further development of the approach is likely to be required for the use of SpiCee as a therapeutic strategy.

In conclusion, we have created a $\mathrm{Ca}^{2+}$ scavenger that has the capability to interfere with $\mathrm{Ca}^{2+}$-dependent physiological functions. This genetically encoded tool has the potential to alter $\mathrm{Ca}^{2+}$ responses in a cell-specific manner and with subcellular resolution, enlarging the possibility of studying local signaling cascades in vivo. Combined with gene therapy strategies and cell-type-specific promoters, SpiCee opens promising perspectives for specific therapeutic interventions targeting $\mathrm{Ca}^{2+}$ with limited side effects compared to pharmacological approaches that do not afford the opportunity for single-cell-type manipulation or specific cellular pathway targeting. 


\section{STAR $\star$ METHODS}

Detailed methods are provided in the online version of this paper and include the following:

- KEY RESOURCES TABLE

- RESOURCE AVAILABILITY

O Lead Contact

O Material Availability

O Data and Code Availability

- EXPERIMENTAL MODEL AND SUBJECT DETAILS

$\bigcirc$ Animals

O HEK293 cell culture

O Retinal explants

- METHOD DETAILS

O Molecular Biology

O Production of purified SpiCee $\mathrm{F}^{\mathrm{F} 134 \mathrm{~W}}$ and SpiCee $\mathrm{F}^{\mathrm{F} 103 \mathrm{~W}}$

○ Measurement of $\mathrm{Ca}^{2+}$ affinity for the labeled site of SpiCee ${ }^{\mathrm{F} 103 \mathrm{~W}}$ (parvalbumin-derived)

- Measurement of $\mathrm{Ca}^{2+}$ affinity for the labeled site of $\mathrm{SpiCee}^{\mathrm{F} 134 \mathrm{~W}}$ (calmodulin-derived)

O Measurement of $\mathrm{Mg}^{2+}$ affinity

O Measurement of kinetic dissociation constants

O Cell death assay

○ Resting $\mathrm{Ca}^{2+}$ concentration measurement

○ Membrane fractionation by detergent-free method

O Analysis of Erk1/2 phosphorylation

$O$ In utero electroporation and mouse brain processing

○ Collapse assay

O Immunostaining

FRET Imaging and analysis

$O$ Imaging of SpiCee targeting

- QUANTIFICATION AND STATISTICAL ANALYSIS

\section{SUPPLEMENTAL INFORMATION}

Supplemental Information can be found online at https://doi.org/10.1016/j. celrep.2020.107934.

\section{ACKNOWLEDGMENTS}

We thank Dr. O. Griesbeck for the gift of Twitch2B. We are grateful to the members of our labs and of the J. Livet lab for thoughtful discussion, to P. Gaspar and N. Spitzer for helpful critical reading of the manuscript, and to the members of the animal and imaging facilities of Institut de la Vision. This work was supported by grants from the ANR (ANR-15-CE16-0007 and ANR-18CE16-0017), Sorbonne Université (FCS-SU IDEX SUPER SU-15-R-PERSU17), and Retina France to X.N. This work was performed in the frame of $L A B E X$ LIFESENSES (reference ANR-10-LABX-65) and of IHU FOReSIGHT (ANR-18IAHU-0001) supported by French state funds managed by the Agence Nationale de la Recherche within the Investissements d'Avenir program. S.B. and A.L. were supported by a fellowships from the ED3C doctoral program (Sorbonne Université).

\section{AUTHOR CONTRIBUTIONS}

Conceptualization, X.N. and O.R.; Methodology, X.N., O.R., S.B., Y.Z., K.L., A.A., C.M., and Y.M.; Validation, X.N., O.R., K.L., and S.B.; Formal Analysis, X.N., O.R., S.B., and Y.M.; Investigation, O.R., S.B., K.L., F.R., S.C., Y.Z., A.A., M.A., A.L., and Y.M.; Writing - Original Draft, O.R. and X.N.; Writing - Review \& Editing, O.R., X.N., K.L., A.L., S.B., S.C., Y.Z., and Y.M.; Visualization,
O.R., S.B., and X.N.; Supervision, X.N., C.M., and C.P.; Project Administration, X.N.; Funding Acquisition, X.N.

\section{DECLARATION OF INTERESTS}

A patent application, listing O.R. and X.N. as inventors, describing the development and applications of SpiCee as a $\mathrm{Ca}^{2+}$ chelator is pending.

Received: October 7, 2019

Revised: May 21, 2020

Accepted: June 29, 2020

Published: July 21, 2020

\section{REFERENCES}

Akiyama, H., Fukuda, T., Tojima, T., Nikolaev, V.O., and Kamiguchi, H. (2016). Cyclic Nucleotide Control of Microtubule Dynamics for Axon Guidance. J. Neurosci. 36, 5636-5649.

Augustine, G.J., Santamaria, F., and Tanaka, K. (2003). Local calcium signaling in neurons. Neuron 40, 331-346.

Averaimo, S., and Nicol, X. (2014). Intermingled cAMP, cGMP and calcium spatiotemporal dynamics in developing neuronal circuits. Front. Cell. Neurosci. 8, 376.

Averaimo, S., Assali, A., Ros, O., Couvet, S., Zagar, Y., Genescu, I., Rebsam, A., and Nicol, X. (2016). A plasma membrane microdomain compartmentalizes ephrin-generated cAMP signals to prune developing retinal axon arbors. Nat. Commun. 7, 12896

Bando, Y., Irie, K., Shimomura, T., Umeshima, H., Kushida, Y., Kengaku, M., Fujiyoshi, Y., Hirano, T., and Tagawa, Y. (2016). Control of Spontaneous Ca2+ Transients Is Critical for Neuronal Maturation in the Developing Neocortex. Cereb. Cortex 26, 106-117.

Clapham, D.E. (2007). Calcium signaling. Cell 131, 1047-1058.

Collatz, M.B., Rüdel, R., and Brinkmeier, H. (1997). Intracellular calcium chelator BAPTA protects cells against toxic calcium overload but also alters physiological calcium responses. Cell Calcium 21, 453-459.

Cooper, D.M.F. (2015). Store-operated $\mathrm{Ca}^{2+}$-entry and adenylyl cyclase. Cell Calcium 58, 368-375.

Depry, C., Allen, M.D., and Zhang, J. (2011). Visualization of PKA activity in plasma membrane microdomains. Mol. Biosyst. 7, 52-58.

Filadi, R., and Pozzan, T. (2015). Generation and functions of second messengers microdomains. Cell Calcium 58, 405-414.

Fukuda, N., Matsuda, T., and Nagai, T. (2014). Optical control of the Ca2+ concentration in a live specimen with a genetically encoded $\mathrm{Ca} 2+-$-releasing molecular tool. ACS Chem. Biol. 9, 1197-1203.

Gifford, J.L., Walsh, M.P., and Vogel, H.J. (2007). Structures and metal-ionbinding properties of the Ca2+-binding helix-loop-helix EF-hand motifs. Biochem. J. 405, 199-221.

Godfraind, T. (2017). Discovery and Development of Calcium Channel Blockers. Front. Pharmacol. 8, 286.

Guan, C.-B., Xu, H.-T., Jin, M., Yuan, X.-B., and Poo, M.-M. (2007). Long-range $\mathrm{Ca} 2+$ signaling from growth cone to soma mediates reversal of neuronal migration induced by slit-2. Cell 129, 385-395.

Guirland, C., Suzuki, S., Kojima, M., Lu, B., and Zheng, J.Q. (2004). Lipid rafts mediate chemotropic guidance of nerve growth cones. Neuron 42, 51-62.

He, M.-L., Zemkova, H., Koshimizu, T.A., Tomić, M., and Stojilkovic, S.S. (2003). Intracellular calcium measurements as a method in studies on activity of purinergic P2X receptor channels. Am. J. Physiol. Cell Physiol. 285, C467C479.

Hutnik, C.M., MacManus, J.P., Banville, D., and Szabo, A.G. (1990). Comparison of metal ion-induced conformational changes in parvalbumin and oncomodulin as probed by the intrinsic fluorescence of tryptophan 102. J. Biol. Chem. 265, 11456-11464. 
Johnson, J.D., and Tikunova, S.B. (2002). Fluorescence methods for measuring calcium affinity and calcium exchange with proteins. Methods Mol. Biol. 173, 89-102.

Kunze, M., and Berger, J. (2015). The similarity between N-terminal targeting signals for protein import into different organelles and its evolutionary relevance. Front. Physiol. 6, 259.

Lee, Y.-H., Tanner, J.J., Larson, J.D., and Henzl, M.T. (2004). Crystal structure of a high-affinity variant of rat alpha-parvalbumin. Biochemistry 43, 1000810017.

Lefkimmiatis, K., Moyer, M.P., Curci, S., and Hofer, A.M. (2009). "cAMP sponge": a buffer for cyclic adenosine 3', 5'-monophosphate. PLoS ONE 4, e7649.

Loulier, K., Lathia, J.D., Marthiens, V., Relucio, J., Mughal, M.R., Tang, S.-C., Coksaygan, T., Hall, P.E., Chigurupati, S., Patton, B., et al. (2009). beta1 integrin maintains integrity of the embryonic neocortical stem cell niche. PLoS Biol. 7, e1000176.

Markwardt, M.L., Kremers, G.-J.J., Kraft, C.A., Ray, K., Cranfill, P.J., Wilson, K.A., Day, R.N., Wachter, R.M., Davidson, M.W., and Rizzo, M.A. (2011). An improved cerulean fluorescent protein with enhanced brightness and reduced reversible photoswitching. PLoS ONE 6, e17896.

May, V., Clason, T.A., Buttolph, T.R., Girard, B.M., and Parsons, R.L. (2014). Calcium influx, but not intracellular calcium release, supports PACAP-mediated ERK activation in HEK PAC1 receptor cells. J. Mol. Neurosci. 54, 342-350.

McMahon, S.M., and Jackson, M.B. (2018). An Inconvenient Truth: Calcium Sensors Are Calcium Buffers. Trends Neurosci. 41, 880-881.

Nagel, G., Szellas, T., Huhn, W., Kateriya, S., Adeishvili, N., Berthold, P., Ollig, D., Hegemann, P., and Bamberg, E. (2003). Channelrhodopsin-2, a directly light-gated cation-selective membrane channel. Proc. Natl. Acad. Sci. USA 100, 13940-13945

Nakai, Y., and Kamiguchi, H. (2002). Migration of nerve growth cones requires detergent-resistant membranes in a spatially defined and substrate-dependent manner. J. Cell Biol. 159, 1097-1108.

Nicol, X., Voyatzis, S., Muzerelle, A., Narboux-Nême, N., Südhof, T.C., Miles, R., and Gaspar, P. (2007). cAMP oscillations and retinal activity are permissive for ephrin signaling during the establishment of the retinotopic map. Nat. Neurosci. 10, 340-347.

Nishiyama, M., Hoshino, A., Tsai, L., Henley, J.R., Goshima, Y., Tessier-Lavigne, M., Poo, M.-M., and Hong, K. (2003). Cyclic AMP/GMP-dependent modulation of $\mathrm{Ca} 2+$ channels sets the polarity of nerve growth-cone turning. Nature 423, 990-995.

Pertusa, J.A.G., Sanchez-Andrés, J.V., Martín, F., and Soria, B. (1999). Effects of calcium buffering on glucose-induced insulin release in mouse pancreatic islets: an approximation to the calcium sensor. J. Physiol. 520, 473-483.

Piazza, M., Taiakina, V., Dieckmann, T., and Guillemette, J.G. (2017). Structural Consequences of Calmodulin EF Hand Mutations. Biochemistry 56, 944-956.

Rajasethupathy, P., Ferenczi, E., and Deisseroth, K. (2016). Targeting neural circuits. Cell 165, 524-534.

Robertson, S.P., Johnson, J.D., and Potter, J.D. (1981). The time-course of $\mathrm{Ca} 2+$ exchange with calmodulin, troponin, parvalbumin, and myosin in response to transient increases in Ca2+. Biophys. J. 34, 559-569.

Ros, O., Zagar, Y., Ribes, S., Baudet, S., Loulier, K., Couvet, S., Ladarre, D., Aghaie, A., Louail, A., Petit, C., et al. (2019). SponGee: A Genetic Tool for Subcellular and Cell-Specific cGMP Manipulation. Cell Rep. 27, 4003-4012.e6.
Rosado, J.A., and Sage, S.O. (2001). Role of the ERK pathway in the activation of store-mediated calcium entry in human platelets. J. Biol. Chem. 276, 1565915665.

Schneider, C.A., Rasband, W.S., and Eliceiri, K.W. (2012). NIH Image to ImageJ: 25 years of image analysis. Nature methods 9, 671-675.

Sengupta, A., Chaffiol, A., Macé, E., Caplette, R., Desrosiers, M., Lampič, M. Forster, V., Marre, O., Lin, J.Y., Sahel, J.-A., et al. (2016). Red-shifted channelrhodopsin stimulation restores light responses in blind mice, macaque retina, and human retina. EMBO Mol. Med. 8, 1248-1264.

Shelly, M., Lim, B.K., Cancedda, L., Heilshorn, S.C., Gao, H., and Poo, M.M. (2010). Local and long-range reciprocal regulation of cAMP and cGMP in axon/dendrite formation. Science 327, 547-552.

Starovasnik, M.A., Su, D.R., Beckingham, K., and Klevit, R.E. (1992). A series of point mutations reveal interactions between the calcium-binding sites of calmodulin. Protein Sci. 1, 245-253.

Tang, W., Halling, D.B., Black, D.J., Pate, P., Zhang, J.-Z., Pedersen, S., Altschuld, R.A., and Hamilton, S.L. (2003). Apocalmodulin and Ca2+ calmodulin-binding sites on the CaV1.2 channel. Biophys. J. 85, 1538-1547.

Thestrup, T., Litzlbauer, J., Bartholomäus, I., Mues, M., Russo, L., Dana, H., Kovalchuk, Y., Liang, Y., Kalamakis, G., Laukat, Y., et al. (2014). Optimized ratiometric calcium sensors for functional in vivo imaging of neurons and T lymphocytes. Nat. Methods 11, 175-182.

Tong, J., McCarthy, T.V., and MacLennan, D.H. (1999). Measurement of resting cytosolic $\mathrm{Ca} 2+$ concentrations and $\mathrm{Ca} 2+$ store size in HEK-293 cells transfected with malignant hyperthermia or central core disease mutant Ca2+ release channels. J. Biol. Chem. 274, 693-702.

Tønnesen, J., and Kokaia, M. (2017). Epilepsy and optogenetics: can seizures be controlled by light? Clin. Sci. (Lond.) 131, 1605-1616.

Tsien, R.Y. (1980). New calcium indicators and buffers with high selectivity against magnesium and protons: design, synthesis, and properties of prototype structures. Biochemistry 19, 2396-2404.

Tsien, R., and Pozzan, T. (1989). Measurement of cytosolic free Ca2+ with quin2. Methods Enzymol. 172, 230-262.

Uchiyama, T., Yoshikawa, F., Hishida, A., Furuichi, T., and Mikoshiba, K. (2002). A novel recombinant hyperaffinity inositol 1,4,5-trisphosphate (IP(3)) absorbent traps IP(3), resulting in specific inhibition of IP(3)-mediated calcium signaling. J. Biol. Chem. 277, 8106-8113.

Uhlén, P., Fritz, N., Smedler, E., Malmersjö, S., and Kanatani, S. (2015). Calcium signaling in neocortical development. Dev. Neurobiol. 75, 360-368.

Wang, W., Barnabei, M.S., Asp, M.L., Heinis, F.I., Arden, E., Davis, J., Braunlin, E., Li, Q., Davis, J.P., Potter, J.D., and Metzger, J.M. (2013). Noncanonical EFhand motif strategically delays $\mathrm{Ca} 2+$ buffering to enhance cardiac performance. Nat. Med. 19, 305-312.

Weeks, K.M., and Crothers, D.M. (1992). RNA binding assays for Tat-derived peptides: implications for specificity. Biochemistry 31, 10281-10287.

Yu, X., Taylor, A.M.W., Nagai, J., Golshani, P., Evans, C.J., Coppola, G., and Khakh, B.S. (2018). Reducing Astrocyte Calcium Signaling In Vivo Alters Striatal Microcircuits and Causes Repetitive Behavior. Neuron 99, 1170-1187.e9.

Zacharias, D.A., Violin, J.D., Newton, A.C., and Tsien, R.Y. (2002). Partitioning of lipid-modified monomeric GFPs into membrane microdomains of live cells. Science 296, 913-916. 


\section{STAR $\star M E T H O D S$}

\section{KEY RESOURCES TABLE}

\begin{tabular}{|c|c|c|}
\hline REAGENT or RESOURCE & SOURCE & IDENTIFIER \\
\hline \multicolumn{3}{|l|}{ Antibodies } \\
\hline Cleaved Caspase 3 (Asp175) & Cell Signaling & $\begin{array}{l}\text { Cat\# 9661S, lot \# } 0043 . \\
\text { RRID: AB_2341188. }\end{array}$ \\
\hline Mouse anti- $\alpha$-tubulin & Sigma-Aldrich & $\begin{array}{l}\text { Cat\# T6199, lot \# 024M4850V. } \\
\text { RRID: AB_477583. }\end{array}$ \\
\hline Rabbit anti- $\beta$-adaptin $(\mathrm{H}-300)$ & Santa Cruz Biotechnology & $\begin{array}{l}\text { Cat\# sc-10762, lot \# E1304. } \\
\text { RRID: AB_2242872 }\end{array}$ \\
\hline Living Colors $\AA^{\circledR}$ DsRed Polyclonal Antibody & Clontech & $\begin{array}{l}\text { Cat\# 632496, lot \# } 1306037 . \\
\text { RRID: AB_10013483 }\end{array}$ \\
\hline Rabbit Anti-Caveolin Polyclonal Antibody & BD Biosciences & $\begin{array}{l}\text { Cat\# 610060, lot \# GR256941. } \\
\text { RRID: AB_397472 }\end{array}$ \\
\hline Phospho-p42/44 Antibody & Cell Signaling & $\begin{array}{l}\text { Cat\# CS_9101, lot\# } 27 \\
\text { RRID: AB_331646 }\end{array}$ \\
\hline p42/44 Antibody & Cell Signaling & $\begin{array}{l}\text { Cat\# CS_4695 } \\
\text { RRID: AB_390779 }\end{array}$ \\
\hline GFP Tag Polyclonal Antibody & Thermo Fisher Scientific & $\begin{array}{l}\text { Cat\# A11122, lot \# } 1789911 . \\
\text { RRID: AB_221569 }\end{array}$ \\
\hline Peroxidase-AffiniPure Goat Anti-Rabbit IgG $(\mathrm{H}+\mathrm{L})$ antibody & Jackson ImmunoResearch Labs & $\begin{array}{l}\text { Cat\# 111-035-003, lot \# } 81283 . \\
\text { RRID: AB_2313567 }\end{array}$ \\
\hline Peroxidase-AffiniPure Goat Anti-Mouse $\lg G(H+L)$ antibody & Jackson ImmunoResearch Labs & $\begin{array}{l}\text { Cat\# 115-035-003. } \\
\text { RRID: AB_10015289 }\end{array}$ \\
\hline $\begin{array}{l}\text { Donkey Anti-Mouse IgG }(\mathrm{H}+\mathrm{L}) \text { Antibody, Alexa Fluor } 488 \\
\text { Conjugated }\end{array}$ & Thermo Fisher Scientific & $\begin{array}{l}\text { Cat\# A21202, lot \# } 1562298 . \\
\text { RRID: AB_141607 }\end{array}$ \\
\hline $\begin{array}{l}\text { Donkey anti-Rabbit IgG }(\mathrm{H}+\mathrm{L}) \text { Highly Cross-Adsorbed } \\
\text { Secondary Antibody, Alexa Fluor } 594\end{array}$ & Thermo Fisher Scientific & $\begin{array}{l}\text { Cat\# A21207, lot \# } 1602780 . \\
\text { RRID: AB_141637 }\end{array}$ \\
\hline \multicolumn{3}{|l|}{ Chemicals, Peptides, and Recombinant Proteins } \\
\hline HRP-conjugated cholera toxin B-subunit & Sigma-Aldrich & Cat\# C3741 \\
\hline Thapsigargin & Sigma-Aldrich & Cat\# T9033, lot\# 125M4070V \\
\hline Adenosine $5^{\prime}$-triphosphate (ATP) disodium salt hydrate & Sigma-Aldrich & Cat\# A1852, lot\# SLBT9665 \\
\hline \multicolumn{3}{|l|}{ Critical Commercial Assays } \\
\hline CellEvent Caspase-3/7 Green Detection Reagent & Thermo Fisher Scientific & $\begin{array}{l}\text { Cat\# C10723, lot \# } 1932830 . \\
\text { RRID: Not available }\end{array}$ \\
\hline \multicolumn{3}{|l|}{ Experimental Models: Cell Lines } \\
\hline HEK293 & ATCC & $\begin{array}{l}\text { Cat\# CRL-1573. } \\
\text { RRID: CVCL_0045 }\end{array}$ \\
\hline \multicolumn{3}{|l|}{ Experimental Models: Organisms/Strains } \\
\hline Mus musculus C57BL/6JRj & Janvier Labs & RRID:MGI:2670020 \\
\hline Mus musculus RjOrl:SWISS & Janvier Labs & RRID:MGI:2168141 \\
\hline \multicolumn{3}{|l|}{ Recombinant DNA } \\
\hline pCX-SpiCee & This Paper & Addgene\# 140836 \\
\hline pCX-Lyn-SpiCee & This Paper & Addgene\# 140837 \\
\hline pCX-SpiCee-Kras & This Paper & Addgene\# 140838 \\
\hline pCX-SpiCee-NLS & This Paper & Addgene\# 140900 \\
\hline pCX-SpiCee-NES & This Paper & Addgene\# 140901 \\
\hline pCX-mutSpiCee & This Paper & Addgene\# 140903 \\
\hline Twitch-2B & Thestrup et al., 2014 & RRID:Addgene_49531 \\
\hline
\end{tabular}




\begin{tabular}{ll}
\hline Continued & \\
\hline REAGENT or RESOURCE & SOURCE \\
\hline Software and Algorithms & \\
\hline ImageJ & Schneider et al., 2012 \\
GraphPad Prism & IDENTIFIER \\
\hline
\end{tabular}

\section{RESOURCE AVAILABILITY}

\section{Lead Contact}

Further information and requests for resources and reagents should be directed to and will be fulfilled by the Lead Contact, Xavier Nicol (xavier.nicol@inserm.fr).

Material Availability

Plasmid generated in this study have been deposited to Addgene under the following name and catalog number: pCX-SpiCee, \#140836; pCX-Lyn-SpiCee, \#140837; pCX-SpiCee-Kras, \#140838; pCX-SpiCee-NLS, \#140900; pCX-SpiCee-NES, \#140901; pCX-mutSpiCee; \#140903.

Data and Code Availability

This study did not generate any unique dataset or code.

\section{EXPERIMENTAL MODEL AND SUBJECT DETAILS}

\section{Animals}

Pregnant C57BL6/J and RjOrl:SWISS mice were purchased from Janvier Labs. All animal procedures were performed in accordance with institutional guidelines and approved by the local ethics committee (C2EA-05: Comité d'éthique en expérimentation animale Charles Darwin). Animals were housed on a $12 \mathrm{~h}$ light/12 h dark cycle. Embryos from dated matings (developmental stage stated in each section describing individual experiments) were not sexed during the experiments and the female over male ratio is expected to be close to 1 .

\section{HEK293 cell culture}

HEK293T cells (ATCC, not authenticated, free of mycoplasma contamination) were maintained in a $37^{\circ} \mathrm{C}, 5 \% \mathrm{CO} 2$ incubator and transfected using Lipofectamine 2000 (Life Technologies) according to the manufacturer's protocol and imaged the day following transfection or fixed and processed for immunocytofluorescence.

\section{Retinal explants}

Retinas of E14 mouse embryos were electroporated with mRFP, Lyn-SpiCee, SpiCee or SpiCee-Kras using two poring pulses (square wave, $175 \mathrm{~V}, 5 \mathrm{~ms}$ duration, with $50 \mathrm{~ms}$ interval) followed by four transfer pulses (40 V, 50 ms and 950 ms interpulse) with a Nepa21 Super Electroporator (NepaGene). Retinas were dissected and kept 24 hours in culture medium (DMEM-F12 supplemented with glutamine (Sigma Aldrich), penicillin/streptomycin (Sigma Aldrich), BSA (Sigma Aldrich) and glucose), in a humidified incubator at $37^{\circ} \mathrm{C}$ and $5 \% \mathrm{CO}$. The day after, they were cut into $200 \mu \mathrm{m}$ squares with a Tissue-Chopper (Mcllwan) and explants were plated on glass coverslips coated with poly-lysine and Laminin (Sigma Aldrich). Cells were cultured for 24 hours in culture medium supplemented with $0.4 \%(\mathrm{w} / \mathrm{v})$ methyl cellulose. All animal procedures were performed in accordance with institutional guidelines and approved by the local ethics committee (C2EA-05: Comité d'éthique en expérimentation animale Charles Darwin). Embryos were not sexed during the experiments and the female over male ratio is expected to be close to 1.

\section{METHOD DETAILS}

Molecular Biology

SpiCee including a FLAG tag in $5^{\prime}$ and a 3 amino acid linker between the parvalbumin and calmodulin moieties underlined in the following sequence (5'-ATGGACTACAAAGACGATGACGACAAGTCCGGAATCGATATGTCGATGACAGACTTGCTCAGCGCTGAG GACATCAAGAAGGCGATAGGAGCCTTIACTGCTGCAGACTCCTTCGACCACAAAAAGTTCTTCCAGATGGTGGGCCTGAAGAAAA AGAGTGCGGATGATGTGAAGAAGGTGTTCCACATTCTGGACAAAGACAAAGATGGCTTCATTGACGAGGATGAGCTGGGGTCCAT TCTGAAGGGCTTCTCCTCAGATGCCAGAGACTTGTCTGCTAAGGAAACAAAGACGCTGATGGCTGCTGGAGACAAGGACGGGGA TGGCAAGATTGGGGTTGAAGAGTTCTCCACTCTGGTGGCCGAAAGCATCGATCTTAAGATGGCTGATCAGCTGACTGAAGAGCAG ATTGCTGAATTTAAGGAGGCTTTCTCCCTATTCGATAAAGATGGTGACGGCACCATCACAACCAAGGAACTGGGGACCGTCATGC 
GGTCACTGGGTCAGAACCCAACAGAAGCCGAGCTGCAGGATATGATCAACGAAGTGGATGCTGATGGCAATGGCACCATTGACTT CCCAGAGTTCTTGACTATGATGGCTAGAAAAATGAAAGACACA-3') was designed in silico in frame with a tandem repeat of the Lyn Kinase $\mathrm{N}$ terminus domain (5'-ATGGGCTGCATCAAGAGCAAGCGCAAGGATGGGCTGCATCAAGAGCAAGCGCAAGGACAAG-3'), and the desired sequences obtained as oligonucleotides from Sigma and Life Technologies, respectively. The oligos were annealed and cloned into pcDNA3-mRFP in frame with the reporter sequence. Lipid-raft-excluded and cytosolic forms of SpiCee were obtained by subcloning into pcDNA3 with or without the CaaX-polylysine motif of Kras (5'-CAAGAAGAAGAAGAAGAAGAAGAGCAA GACCAAGTGCGTGATCATG-3'), respectively. For expression in retinal ganglion cells, the constructs were subcloned into pCX. Twitch2B was obtained from Addgene and subcloned into pcDNA3. SpiCee-C (5'-GCTGATCAGCTGACTGAAGAGCAGATTGCT GAATTTAAGGAGGCTTTCTCCCTATTCGATAAAGATGGTGACGGCACCATCACAACCAAGGAACTGGGGACCGTCATGCGGTCAC TGGGTCAGAACCCAACAGAAGCCGAGCTGCAGGATATGATCAACGAAGTGGATGCTGATGGCAATGGCACCATTGACTTCCCAGA GTTCTTGACTATGATGGCTAGAAAAATGAAAGACACACTTAAGGCGGATCCCGCCACC-3') was generated by digesting pCXSpiCee with the restriction enzyme Clal.

Production of purified SpiCee ${ }^{\mathrm{F} 134 \mathrm{~W}}$ and SpiCee ${ }^{\mathrm{F} 103 \mathrm{~W}}$

The full-length SpiCee DNA was subcloned into a pGST-Parallel2 vector (derived from pGEX-4T-1; Amersham) in frame with the N-terminal glutathione S-transferase (GST) tag. SpiCee ${ }^{\mathrm{F} 134 \mathrm{~W}}$ and SpiCee ${ }^{\mathrm{F} 103 \mathrm{~W}}$ clones were prepared from SpiCee using the QuickChange II Site-directed mutagenesis kit (Agilent Technologies). Proteins were produced in BL21 (DE3) pLysS E. coli cells. The GSTtagged proteins were purified using a glutathione Sepharose 4B column. The GST tag was cleaved using recombinant tobacco etch virus Nla proteinase. The recombinant proteins were eluted in a buffer containing $30 \mathrm{mM}$ MOPS (3-Morpholinopropanesulfonic acid), $\mathrm{pH} 7.2$ and $100 \mathrm{mM} \mathrm{KCl}$. Protein concentrations were deduced from $\mathrm{A}_{280}$ measurements using a computed extinction coefficient of $5500 \mathrm{M}^{-1} \mathrm{~cm}^{-1}$ for both SpiCee ${ }^{\mathrm{F} 134 \mathrm{~W}}$ and SpiCee ${ }^{\mathrm{F} 103 \mathrm{~W}}$.

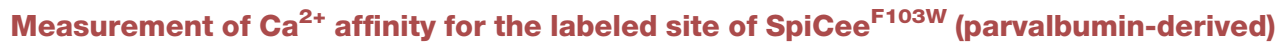
$\mathrm{Ca}^{2+}$ binding to the labeled site of SpiCee ${ }^{\mathrm{F} 103 \mathrm{~W}}$ was monitored by measuring tryptophan fluorescence $(285 \mathrm{~nm}$ excitation, $330 \mathrm{~nm}$ emission, $5 \mathrm{~nm}$ bandpass) at $20^{\circ} \mathrm{C}$ in a $4 \mathrm{~mm} \times 10 \mathrm{~mm}$ quartz cuvette (Jasco FP-8300 spectrofluorimeter). The protein was diluted to $2.25 \mu \mathrm{M}$ in standard buffer $(30 \mathrm{mM}$ MOPS- $\mathrm{KOH} \mathrm{pH} 7.2 ; 0.1 \mathrm{M} \mathrm{KCl})$ and $254 \mu \mathrm{L}$ samples were loaded in the cuvette. It was first observed that addition of $\mathrm{CaCl}_{2}$ did not increase fluorescence, whereas addition of millimolar amounts of EGTA led to large decreases (up to $27 \%$ at a final EGTA concentration of $7.5 \mathrm{mM}$ ). This showed that the labeled site of SpiCee ${ }^{\mathrm{F} 103 \mathrm{~W}}$ was saturated with $\mathrm{Ca}^{2+}$. Dissociation constant of $\mathrm{Ca}^{2+}$ was deduced from a competition experiment in which the protein solution was titrated with EGTA. $2 \mu \mathrm{L}$ samples of solutions containing increasing concentrations of EGTA (1.95 to $500 \mathrm{mM}$ ) were added to the cuvette. Fluorescence was measured after each addition. Fluorescence values were corrected for dilution and plotted as a function of the concentration of EGTA. Data were fitted to the quadratic Equation 1 as described (Weeks and Crothers, 1992), assuming that the two parvalbumin-derived sites were equivalent for $\mathrm{Ca}^{2+}$ binding.

$$
F=F_{0}+\left(F_{\infty}-F_{0}\right) \frac{\left[K_{D}+K_{E G T A}[E G T A]+[\mathrm{Ca}]+[P]-\sqrt{\left(K_{D}+K_{E G T A}[E G T A]+[\mathrm{Ca}]+[P]\right)^{2}-4[P][\mathrm{Ca}]}\right]}{2[P]}
$$

(Equation 1)

where $F_{0}$ is the fluorescence of the $\mathrm{Ca}^{2+}$-saturated protein, $\mathrm{F}_{\infty}$ the fluorescence of $\mathrm{Ca}^{2+}$-free protein, $[\mathrm{P}]$ the concentration in highaffinity $\mathrm{Ca}^{2+}$-binding sites (here $4.5 \mu \mathrm{M}$ ), $\mathrm{K}_{\mathrm{EGTA}}$ the dissociation constant of $\mathrm{Ca}^{2+}$ from its complex with EGTA, $K_{\mathrm{D}}$ the dissociation constant of $\mathrm{Ca}^{2+}$ from its complex with the parvalbumin site, $[\mathrm{Ca}]$ the total concentration of $\mathrm{Ca}^{2+}$ and [EGTA] the total concentration of EGTA.

$K_{D}, F_{0}$, and $F_{\infty}$ and their associated standard errors were derived from non-linear least square fitting with the Origin 9.0 software. Results are the mean \pm standard deviation from three independent experiments. Fitting required the input of two values. The dissociation constant of $\mathrm{Ca}^{2+}$ from its complex with EGTA is $0.15 \mu \mathrm{M}$ in these conditions (Tsien and Pozzan, 1989). The total concentration of $\mathrm{Ca}^{2+}$ in the sample was calculated as the concentration of $\mathrm{Ca}^{2+}$ bound to the two parvalbumin sites (i.e., $\left.4.5 \mu \mathrm{M}\right)$ plus the concentration of free $\mathrm{Ca}^{2+}$ and the calmodulin-derived sites of SpiCee bound to $\mathrm{Ca}^{2+}$. The latter value was measured using the fluorescent calcium indicator Quin-2 (50 $\mu \mathrm{M}$; excitation $332 \mathrm{~nm}$, emission $493 \mathrm{~nm})$. At the concentrations used, the indicator was not sensitive to the parvalbumin-derived sites of SpiCee bound to $\mathrm{Ca}^{2+}$ because of its much lower affinity for $\mathrm{Ca}^{2+}(60 \mathrm{nM})$ (Tsien and Pozzan, 1989). A value of $2 \mu \mathrm{M}$ for the concentration of free plus calmodulin-derived sites-bound to $\mathrm{Ca}^{2+}$ was computed from this experiment. Therefore, a total concentration of $\mathrm{Ca}^{2+}$ of $6.5 \mu \mathrm{M}$ was used for fitting using Equation 1.

Measurement of $\mathrm{Ca}^{2+}$ affinity for the labeled site of SpiCee ${ }^{\mathrm{F} 134 \mathrm{~W}}$ (calmodulin-derived) $\mathrm{Ca}^{2+}$ binding to the first calmodulin site of SpiCee ${ }^{\mathrm{F} 134 \mathrm{~W}}$ was monitored by measuring tryptophan fluorescence (285 $\mathrm{nm}$ excitation, $340 \mathrm{~nm}$ emission, $5 \mathrm{~nm}$ bandpass) at $20^{\circ} \mathrm{C}$. SpiCee ${ }^{\mathrm{F} 134 \mathrm{~W}}$ was diluted to $1.6 \mu \mathrm{M}$ in standard buffer and $258 \mu \mathrm{L}$ samples were loaded in the cuvette. Addition of $\mathrm{CaCl}_{2}$ increased fluorescence, whereas addition of millimolar amounts of EGTA led to fluorescence decrease, showing that the calmodulin-derived sites of SpiCee were partially filled with $\mathrm{Ca}^{2+}$ in the sample. The value of the fluorescence of the protein with $\mathrm{Ca}^{2+}$-free calmodulin sites was measured in the presence of $2 \mathrm{mM}$ EGTA, a concentration found to be 
saturating. Total concentration of $\mathrm{Ca}^{2+}$ in the sample (excluding $\mathrm{Ca}^{2+}$ bound to the parvalbumin-derived sites of SpiCee) was measured as described above using Quin-2 and found to be $2.5 \mu \mathrm{M}$. Dissociation constant of $\mathrm{Ca}^{2+}$ from the labeled site of SpiCe$\mathrm{e}^{\mathrm{F} 134 \mathrm{~W}}$ was deduced from an experiment where the protein solution was titrated with increasing amounts of $\mathrm{CaCl}_{2}(2 \mu \mathrm{L}$ additions of solutions from 0.05 to $6.25 \mathrm{mM}$ ). Fluorescence values were corrected for dilution, and plotted as a function of total $\mathrm{Ca}^{2+} \mathrm{concentration}$ (added $\mathrm{Ca}^{2+}$ plus $2.5 \mu \mathrm{M}$ ). Data were fitted to a binding curve (equ. 2) assuming that the two calmodulin sites were equivalent for $\mathrm{Ca}^{2+}$ binding.

$$
F=F_{0}+\frac{\left(F_{\infty}-F_{0}\right)\left[[P]+[\mathrm{Ca}]+K_{D}-\sqrt{\left([P]+[\mathrm{Ca}]+K_{D}\right)^{2}-4[P][\mathrm{Ca}]}\right]}{2[P]}
$$

where $\mathrm{F}_{0}$ is the fluorescence of the $\mathrm{Ca}^{2+}$-free protein, $\mathrm{F}_{\infty}$ the fluorescence of $\mathrm{Ca}^{2+}$-saturated protein, [P] the concentration in lowaffinity $\mathrm{Ca}^{2+}$-binding sites (here $3.2 \mu \mathrm{M}$ ), $K_{\mathrm{D}}$ the dissociation constant of $\mathrm{Ca}^{2+}$ from its complex with the labeled site of SpiCee ${ }^{\mathrm{F} 134 \mathrm{~W}}$, [Ca] the total concentration of $\mathrm{Ca}^{2+}$.

Measurement of $\mathrm{Mg}^{2+}$ affinity

Titrations with $\mathrm{Mg}^{2+}$ were made in the presence of $10 \mathrm{mM}$ EGTA (F103W) or 2 mM EGTA (F134W) to remove Ca ${ }^{2+}$ from the studied sites. The concentrations of free $\mathrm{Mg}^{2+}$ during titration were calculated using a dissociation constant of $19.73 \mathrm{mM}$ for the $\mathrm{Mg}^{2+}: \mathrm{EGTA}^{2}$ complex (Tsien and Pozzan, 1989).

$K_{D}, F_{0}$, and $F_{\infty}$ and their associated standard errors were derived from non-linear least square fitting with the Origin software (Origin Lab.). Results are the mean \pm standard deviation from three independent experiments.

\section{Measurement of kinetic dissociation constants}

Values of kinetic dissociation constants $\left(k_{\text {off }}\right)$ for the tryptophan-labeled Ca binding sites of SpiCee (SpiCee ${ }^{\mathrm{F} 103 \mathrm{~W}}$ and SpiCee ${ }^{\mathrm{F} 134 \mathrm{~W}}$ ) were measured by mixing proteins with appropriate amounts of EGTA at $20^{\circ} \mathrm{C}$ in an Applied Photophysics stopped flow apparatus. Reacting solutions were excited at $285 \mathrm{~nm}$ and fluorescence was measured using a photomultiplier equipped with a $320 \mathrm{~nm}$ cutoff filter. For SpiCee ${ }^{\mathrm{F} 103 \mathrm{~W}}$, the protein $\left(2.3 \mu \mathrm{M}\right.$ in standard buffer) was mixed 1:1 with $15 \mathrm{mM}$ EGTA in the same buffer. For SpiCee $\mathrm{F}^{\mathrm{F} 134 \mathrm{~W}}$, the protein $\left(1.7 \mu \mathrm{M}\right.$ in standard buffer plus $\left.50 \mu \mathrm{M} \mathrm{CaCl}_{2}\right)$ was mixed 1:1 with $4 \mathrm{mM}$ EDTA in standard buffer plus $50 \mu \mathrm{M} \mathrm{CaCl}$. Fluorescence was recorded as a function of time and data were fitted with single exponential curves from which rate constants were derived. Reported values are the mean \pm standard deviation from three measurements.

\section{Cell death assay}

HEK293 Cells were plated on poly-lysine-coated coverslips and transfected the following day with a pCX-mRFP or a pCX-SpiCee vector using Lipofectamine 2000 (Thermo Fisher) following the manufacturer's instructions. Retinas of E14 mouse embryos were electroporated with mRFP or SpiCee using two poring pulses (square wave, $175 \mathrm{~V}, 5$ ms duration, with 50 ms interval) followed by four transfer pulses ( $40 \mathrm{~V}, 50 \mathrm{~ms}$ and $950 \mathrm{~ms}$ interpulse) with a Nepa21 Super Electroporator (NepaGene). Retinas were dissected and kept 24 hours in culture medium (DMEM-F12 supplemented with glutamine (Sigma Aldrich), penicillin/streptomycin (Sigma Aldrich), BSA (Sigma Aldrich) and glucose), in a humidified incubator at $37^{\circ} \mathrm{C}$ and $5 \% \mathrm{CO} 2$. The day after, they were cut into $200 \mu \mathrm{m}$ squares with a Tissue-Chopper (Mcllwan) and explants were plated on glass coverslips coated with poly-lysine and Laminin (Sigma Aldrich). Cells were cultured for 24 hours in culture medium supplemented with $0.4 \%(\mathrm{w} / \mathrm{v})$ methyl cellulose.

Three days (HEK293 cells) or 24h (retinal explants) after plating, cells were either fixed with 4\% (w/v) paraformaldehyde and processed for immunocytochemistry with the antibodies against cleaved caspase 3 (Asp175; Cell Signaling; lot \# 0043) and DsRed (Ozyme), or treated with the CellEvent Caspase 3/7 Green Detection Reagent (Thermo Fisher) for 30 minutes and then fixed and labeled with an $\alpha$-DsRed (Ozyme) antibody. For each experiment, the proportion of caspase3-positive over unlabeled cells in the population of mRFP- or SpiCee-positive cells was computed from 10 randomly chosen fields acquired using a 20x air objective in a DM6000 microscope (Leica Microsystems) (HEK293 cells) or from one optical section of the retinal explant acquired using a 40x dry objective in a FV1000 confocal microscope (Olympus) (retinal explants).

\section{Resting $\mathrm{Ca}^{2+}$ concentration measurement}

HEK293 Cells were plated on poly-lysine-coated $35 \mathrm{~mm}$ plates and transfected 24 hours later with a pCX-mRFP or a pCX-SpiCee vector using Lipofectamine 2000 (Thermo Fisher) and the recommended protocol. The following day, cells were loaded with Fura-2AM (Thermo Fisher) for 1 hour at $37^{\circ} \mathrm{C}$, washed thrice and bathed in HBS with either 0.2 or $2 \mathrm{mM} \mathrm{CaCl}$. Randomly chosen fields were acquired with a 40x water immersion objective (N.A. 0.8) in an Eclipse 80i microscope (Nikon). Fura-2 fluorescence from 340 and $380 \mathrm{~nm}$ excitation was acquired sequentially with the software Imaging Workbench 6.0 (INDEC Biosystems) controlling a Lambda DG-4 (Sutter Instruments). The emission at $594 \mathrm{~nm}$ was also acquired to verify the expression of mRFP and SpiCee.

Calcium concentration was assessed by computing the ratio of emissions obtained from the $340 \mathrm{~nm}$ and $380 \mathrm{~nm}$ excitation. Images were corrected for background fluorescence and signal induced by the mRFP bleedthrough in each of the wavelengths before ratio 
calculation. Such ratio was then converted into calcium concentration using a calibration curve obtained with a Fura-2 Calcium Imaging Calibration Kit imaged in the same experimental conditions (Thermo Fisher).

\section{Membrane fractionation by detergent-free method}

Electroporated retinas were pelleted $\left(195 \mathrm{~g}\right.$ for $5 \mathrm{~min}$ at $\left.4^{\circ} \mathrm{C}\right)$ and resuspended in $1.34 \mathrm{~mL}$ of $0.5 \mathrm{M}$ sodium carbonate, $\mathrm{pH} 11.5$, with protease inhibitor cocktail and phosphatase inhibitor cocktails 1, 2 and 3 (Sigma-Aldrich). The homogenate was sheared through a 26-gauge needle and sonicated three times for $20 \mathrm{~s}$ bursts. The homogenate was adjusted to $40 \%$ (w/v) sucrose by adding $2.06 \mathrm{~mL}$ of $60 \%(\mathrm{w} / \mathrm{v})$ sucrose in MBS (25 mM MES, pH 6.4, $150 \mathrm{mM} \mathrm{NaCl}$, and $250 \mathrm{mM}$ sodium carbonate), placed under a 5\%-30\% (w/v) discontinuous sucrose gradient, and centrifuged at 34,000 rpm for $15-18 \mathrm{~h}$ at $4{ }^{\circ} \mathrm{C}$ in a Beckman SW $41 \mathrm{Ti}$ rotor. Nine fractions (1.24 mL each) were harvested from the top of the tube mixed with 9 volumes of MBS, and centrifuged at $40,000 \mathrm{rpm}$ for $1 \mathrm{~h}$ at $4^{\circ} \mathrm{C}$ (Beckman SW-41Ti rotor). Supernatants were discarded, and membrane pellets were resuspended in $100 \mu \mathrm{L}$ of $1 \%$ (w/v) SDS.

For immunoblotting, samples were separated on a precast gel (4\%-15\% Mini- Protean TGX Tris-Glycine-buffer SDS-PAGE, Biorad) and transferred onto $0.2 \mu \mathrm{m}$ Trans-Blot Turbo nitrocellulose membranes (Biorad). Membranes were blocked for one hour at room temperature in 1xTBS $(10 \mathrm{mM}$ Tris $\mathrm{pH} 8.0,150 \mathrm{mM} \mathrm{NaCl})$ supplemented with $5 \%(\mathrm{w} / \mathrm{v})$ dried skim milk powder. Primary antibody incubation was carried out overnight at $4{ }^{\circ} \mathrm{C}$, with the following antibodies: rabbit anti-DsRed (1/200; 632476; Clontech; lot \# 1306037), rabbit anti- $\beta$-Adaptin (1/200; sc-10762; Santa Cruz; lot \# E1304) and rabbit anti-Caveolin (1/500; 610060; BD Transduction Laboratories; lot \# GR256941-5). All primary antibodies have been previously validated for this assay (Averaimo et al., 2016). A goat anti-rabbit-HRP-coupled secondary antibody was used for detection (Jackson ImmunoResearch, West Grove, PA). After antibody incubations, membranes were extensively washed in TBS T (TBS containing 2.5\% (v/v) Tween-20). Western blots were visualized using the enhanced chemiluminescence method (ECL prime Western Blotting detection reagent, Amersham).

For dot blot analysis (CtB staining), $3 \mu \mathrm{L}$ of each fraction, resuspended in MBS, were blotted on nitrocellulose membrane, dried for $1 \mathrm{~h}$ and blocked for $1 \mathrm{~h}$ with $3 \%(\mathrm{w} / \mathrm{v})$ dried skim milk powder at room temperature. The membrane was incubated overnight with HRP-conjugated cholera toxin B-subunit (Sigma Aldrich C3741) and detected with ECL Prime.

\section{Analysis of Erk1/2 phosphorylation}

For phosphorylation studies, HEK293T cells were transfected or not with SpiCee and/or Twitch2B vectors for 24 hours after plating using Lipofectamine 2000 according to the manufacturer's instructions (Invitrogen). For stimulation, cells were incubated for 5 minutes with $1 \mu \mathrm{M}$ Thapsigargin or vehicle at $37^{\circ} \mathrm{C}$ in the presence or not of BAPTA. In this case, 30 minutes before stimulation with BAPTA-AM $50 \mu \mathrm{M}$ or vehicle solution was applied. Thapsigargin stimulation reaction was stopped by placing cells on ice followed by extraction of proteins described before. Samples were then separated and membranes incubated with a rabbit anti-phosphoERK1/2 (Thr202/Tyr204) (1/500; CS_9101; Cell Signaling) or a rabbit anti-ERK1/2 (1/1000; CS_4695. Cell Signaling) and visualized with goat anti-rabbit HRP as secondary antibodies and ECL Prime for the detection.

In utero electroporation and mouse brain processing

Timed pregnant females (Janvier Labs) were delivered to Institute's animal facility a week prior to the surgery in order to allow a minimum of 5-day adaptation period. In utero electroporation was performed as described previously (Loulier et al., 2009). E14.5 timed pregnant females were anesthetized with Ketamine/Xylazine and a midline laparotomy was performed, exposing uterine horns and allowing visualization of embryos under oblique illumination. $1 \mu \mathrm{L}$ of DNA containing two plasmid vectors combined 3:1 with sterile Fast Green dye (Sigma) was injected with a glass capillary pipette (75-125 $\mu \mathrm{m}$ outer diameter with beveled tip) driven by a INJECT+MATIC (INJECT+MATIC) microinjector into the lateral ventricle of each embryo. A plasmid encoding green fluorescent protein under the control of the chicken beta actin promoter (pCX-eGFP) was used to monitor the success of electroporation. The second plasmid encoded either a red fluorescent protein (mRFP) control construct or SpiCee-mRFP. The anode of 5 mm diameter tweezertrodes (Sonidel Limited) was placed above the dorsal telencephalon and four $35 \mathrm{~V}$ pulses of $50 \mathrm{~ms}$ duration were applied across the uterine sac. Following intrauterine surgery, the incision site was closed with sutures (4-0, Ethicon) and the mouse was allowed to recover in a clean cage. Mice were either euthanized 4 days after surgery to harvest E18.5 embryonic brains, or allowed to give birth for analysis at P10. Embryonic brains were dissected out, immersed overnight in Antigenfix (Diapath) fixative solution and rinsed in PBS prior to sectioning. P10 mice were deeply anesthetized with sodium pentobarbital $\left(150 \mathrm{mg} \cdot \mathrm{kg}^{-1}\right)$, perfused transcardially with Antigenfix (Diapath), brains dissected out and postfixed overnight in the same solution. Embryonic and postnatal brain samples were sectioned at $200 \mu \mathrm{m}$ thickness on a vibrating blade microtome (Leica VT 1000S) and, sections were either mounted in Vectashield+Dapi (Vector laboratories) or incubated 2 hours in $10 \mu \mathrm{g} \cdot \mathrm{mL}^{-1}$ bis-benzimide (Sigma) and mounted in Mowiol 4-88 (Sigma Aldrich). Confocal images were acquired with a $10 x$ objective (N.A. 0.4) and a Z stack containing the whole specimen was sampled at Nyquist frequency. Images were rendered in ImageJ and Photoshop.

\section{Collapse assay}

Retinal explants were treated with $200 \mathrm{ng} \cdot \mathrm{mL}^{-1} \mathrm{rmSlit}-1$ or $500 \mathrm{ng} \cdot \mathrm{mL}^{-1} \mathrm{rmEphrinA} 5$ (R\&D Systems) for 1 hour before fixation with $4 \%$ (w/v) PFA in PB for 30 minutes. 
Immunostaining

Retinal explants, or HEK cells coexpressing the targeted versions of SpiCee and GFP, were fixed with $4 \%$ (w/v) PFA in PB for 30 minutes, permeabilized and blocked with $1 \%(\mathrm{v} / \mathrm{v})$ Triton and 3\% (w/v) BSA in PBS, then immunized against DsRed (Clontech, lot \# 1306037, previously used in a similar assay (Averaimo et al., 2016)) followed by a secondary antibody coupled to AlexaFluor 594 (Life Technologies) and GFP (Life Technologies, lot \# 1789911, previously validated (Nicol et al., 2007)) or $\alpha$-Tubulin (Sigma, lot \# 024M4850V, validated (Nicol et al., 2007)) followed by a secondary antibody coupled to AlexaFluor 488 (Life Technologies).

FRET Imaging and analysis

Images were acquired with an inverted DMI6000B epifluorescence microscope (Leica) coupled to a 40x oil-immersion objective (N.A. 1.3) and Metamorph software (Molecular Devices). For live imaging experiments, cells transfected with Twitch2B, and co-expressing mRFP, SpiCee or its variants were perfused with $\mathrm{HBS}$ buffer with 0.2 or $2 \mathrm{mM} \mathrm{CaCl}_{2}$. Thapsigargin (Sigma-Aldrich) was used at $1 \mu \mathrm{M}$; ATP (Sigma-Aldrich) was used at $5 \mu \mathrm{M}$ in HBS $1.5 \mathrm{mM} \mathrm{CaCl}_{2}$. Images were acquired simultaneously for the CFP (483/32 nm) and YFP $(542 / 27)$ channels every $20 \mathrm{~s}$, or every $5 \mathrm{~s}$ for ATP stimulation, while cells were continuously superfused with the medium described above. Simultaneous CFP and YFP channel acquisition was achieved using a dual chip CCD camera ORCA-D2 (Hamamatsu). The wavelength used for CFP excitation in HEK cells was $436 / 20 \mathrm{~nm}$. Images were processed in ImageJ, corrected for background and bleedthrough from CFP into the YFP channel, and the ratio CFP/YFP was computed and normalized to the initial values for each single cell. For better evaluation of the cell morphology, the YFP channel (not the image of the ratio) is illustrated in all figures describing FRET experiments.

\section{Imaging of SpiCee targeting}

Confocal images were acquired with a $63 x$ oil immersion objective (N.A. 1.45) and a Z stack containing the whole specimen was sampled at nyquist frequency in an Olympus confocal microscope (FV1000). Images were rendered in ImageJ and Photoshop.

\section{QUANTIFICATION AND STATISTICAL ANALYSIS}

No data were excluded from the analysis. No sample size calculation was performed. Sample size was considered sufficient after three reproducible and independent experiments, leading to $n \geq 3$ since several animals, coverslips, or biochemical assays were often analyzed for the same experimental condition. Animals or cultures were equivalent and not distinguishable one from another before treatment, de facto randomizing the sample without the need of a formal randomization process. Photomicrographs were often easily traceable by eye to its experimental condition, making blind analysis of the data difficult to achieve. When careful blinding was performed, experiments reproduced the results obtained in non-blinded experiments with identical experimental conditions. Image calculation and analysis were performed using ImageJ.

Statistical tests were calculated using GraphPad Prism (GraphPad Software Inc.). Table S1 summarizes the tests used, $P$ values and number of replicates for all the data shown in Figures 1, 2, 3, 4, 5, and 6 and Figures S1, S2, S3, S4, S5, and S6. 
Cell Reports, Volume 32

Supplemental Information

SpiCee: A Genetic Tool for Subcellular

and Cell-Specific Calcium Manipulation

Oriol Ros, Sarah Baudet, Yvrick Zagar, Karine Loulier, Fiona Roche, Sandrine Couvet, Alain Aghaie, Melody Atkins, Alice Louail, Christine Petit, Christine Metin, Yves Mechulam, and Xavier Nicol 


\section{SUPPLEMENTARY FIGURES}

A
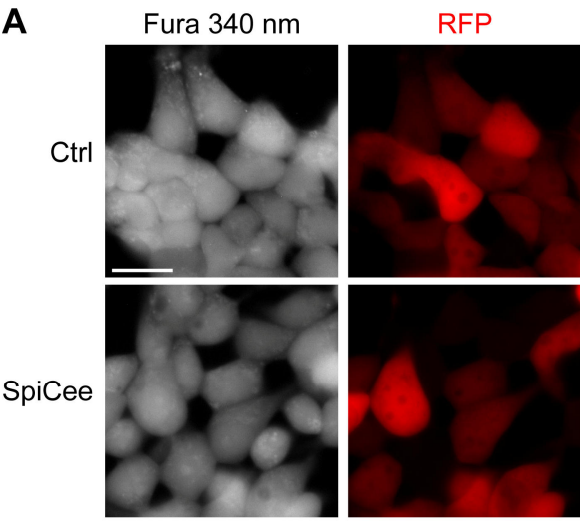

$340 / 380$ ratio

B

Fura $340 \mathrm{~nm}$

RFP
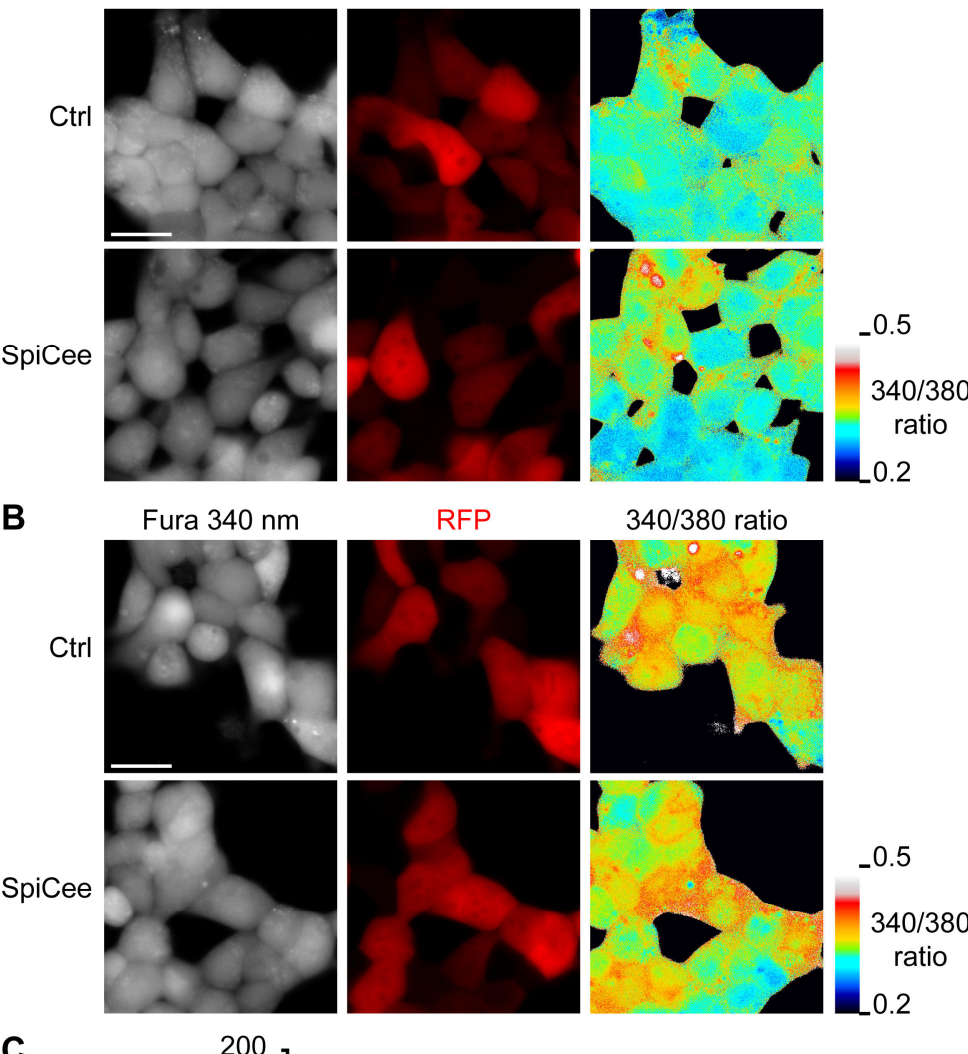

$340 / 380$ ratio

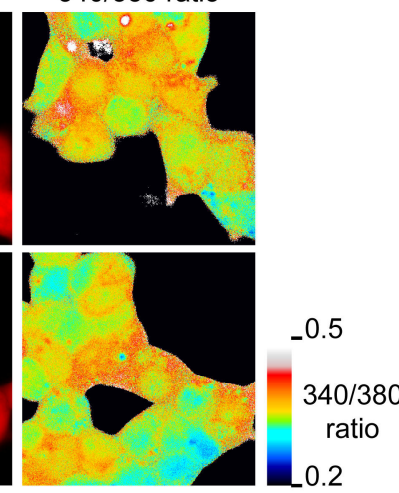

C

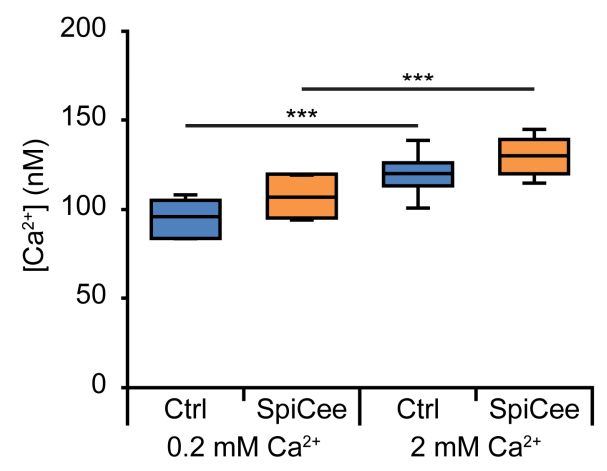

Figure S1. SpiCee-transfected cells adapt to maintain a resting concentration of $\mathrm{Ca}^{2+}$ similar to their controls. Related to Figure 2. SpiCee- or RFP-transfected HEK293 cells were loaded with the ratiometric calcium sensor Fura-2, enabling to evaluate their intracellular calcium concentration. (A) Fura-2 was sequentially excited at $340 \mathrm{~nm}\left(\mathrm{Ca}^{2+}\right.$-bound) and $380 \mathrm{~nm}\left(\mathrm{Ca}^{2+}\right.$-free $)$, and the intracellular $\mathrm{Ca}^{2+}$ concentration was computed based on the fluorescence of calibrated $\mathrm{Ca}^{2+}$ solutions. No difference in the resting $\mathrm{Ca}^{2+}$ concentration was detected between SpiCee- and RFP-transfected cell in culture media containing either (A) $0.2 \mathrm{mM}$ or (B) $2 \mathrm{mM} \mathrm{Ca}^{2+}$. (C) The $\mathrm{Ca}^{2+}$ resting concentration is higher in both SpiCee- and RFPtransfected cells grown in $2 \mathrm{mM}$ extracellular $\mathrm{Ca}^{2+}$ as compared to cells kept in $0.2 \mathrm{mM} \mathrm{Ca}^{2+}$, suggesting that SpiCee does not prevent the regulation of the intracellular resting concentration of this ion. (A, B) Scale bar, $20 \mu \mathrm{m}$. The scale bar in (B) applies to (A). (C) Box-and-whisker plot elements: center line, mean; box limits, upper and lower quartiles; whiskers, s.d.; *** $P \leq 0.001$, Kruskal-Wallis test followed by Dunn's multiple comparison test. Exact $P$ values and number of replicates are provided in Table S1. 
A

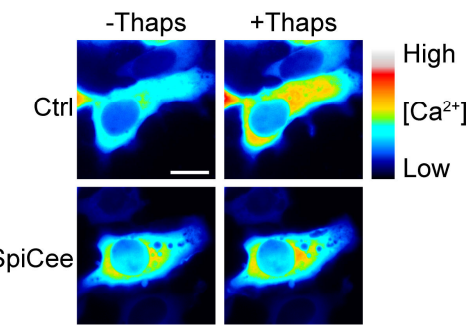

D

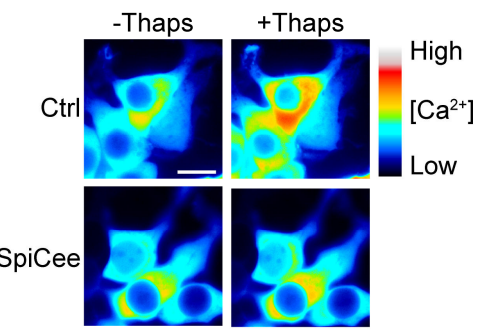

G

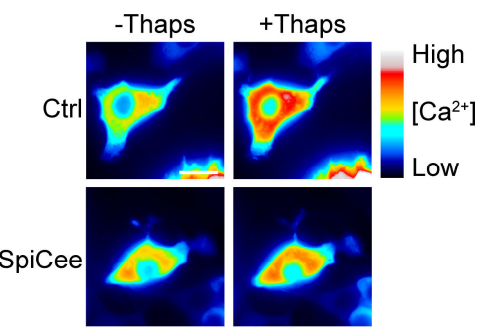

J

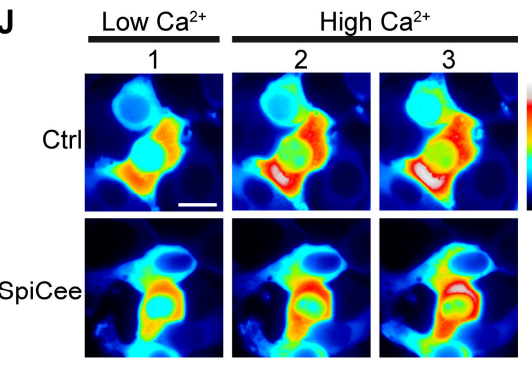

M

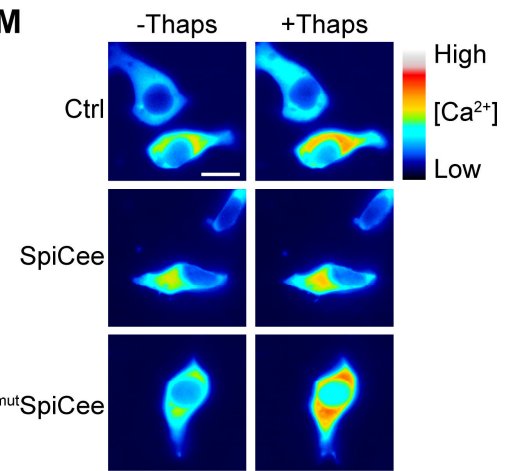

B

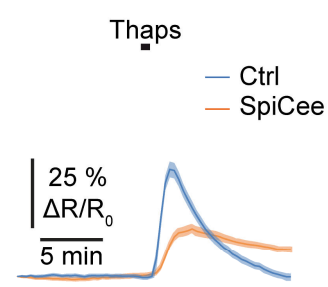

E

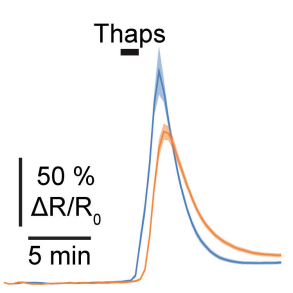

H

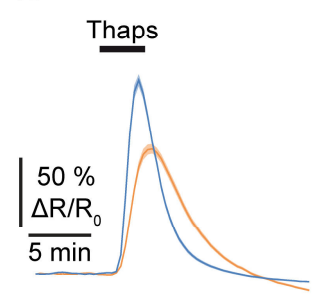

K

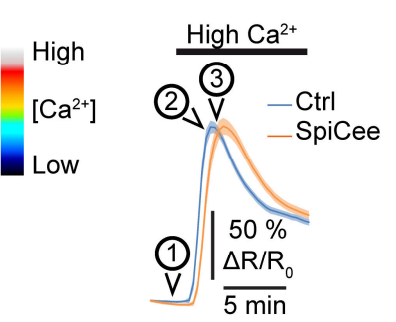

$\mathbf{N}$

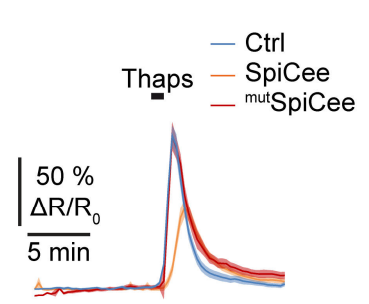

C

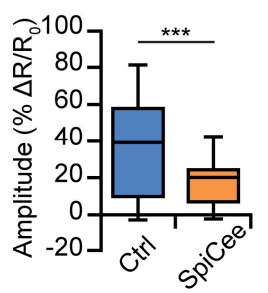

$\mathbf{F}$

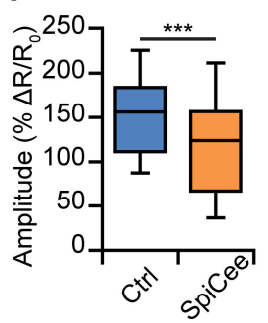

I

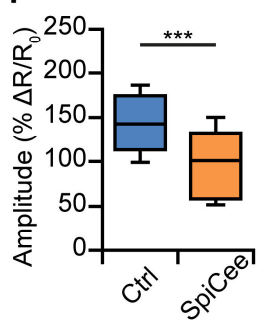

L

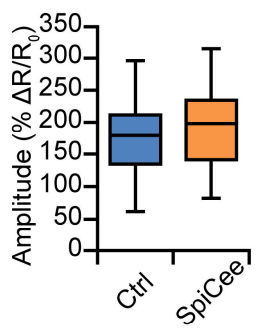

0

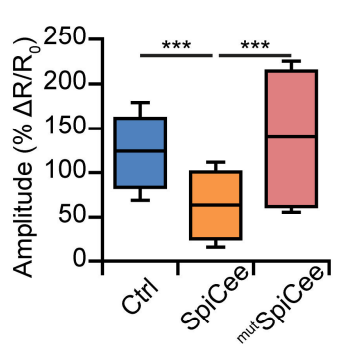

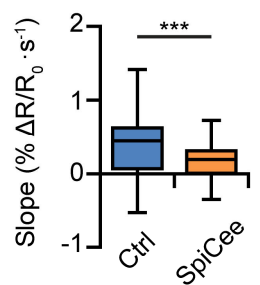
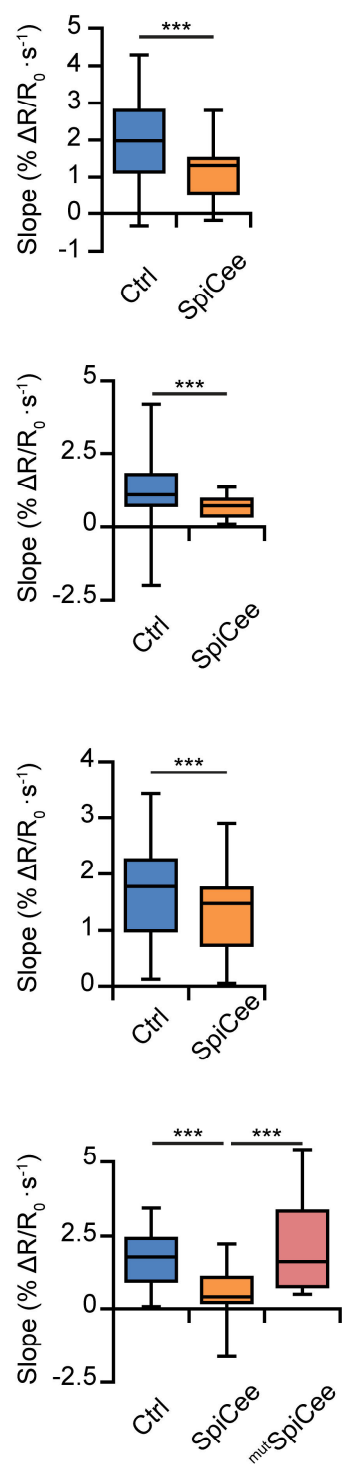

Figure S2. SpiCee reduces the elevation of $\mathrm{Ca}^{2+}$ concentration induced by pulses of thapsigargin and delays massive and sustained elevations of intracellular $\mathrm{Ca}^{2+}$ concentration. Related to Figure 3. HEK293 cells were bathed with a low $\mathrm{Ca}^{2+}$ medium and challenged with a 1-minute (A-C) (See also Video S2), 2-minute (D-F) (See also Video S3) and 5-minute (G-I) (See also Video S4) pulse of thapsigargin. SpiCee-expressing cells display a reduced FRET/CFP ratio elevation compared to controls, and a delayed maximum response. The decay of the response is also delayed in SpiCee-expressing cells, reducing the sharpness of the response. (J) A change of extracellular $\mathrm{Ca}^{2+}$ concentration (from $0.2 \mathrm{mM}$ to $2 \mathrm{mM}$ ) 
generates a massive elevation of fluorescence in the Twitch2B FRET channel in HEK293 cells. The numbers on top of each column reflect the time of acquisition for each image and match the position of the numbered time point in panel K. (K, L) SpiCee-expressing cells exhibit a change in the FRET/CFP ratio of similar magnitude, but delayed as compared to cells devoid of SpiCee (See also Video S5). (M) ${ }^{\mathrm{mut}}$ SpiCee, a variant in which the calcium binding sites have been mutated, does not prevent the elevation of fluorescence in the Twitch2B FRET channel induced by a 1-minute pulse of thapsigargin. (N, O) The FRET/CFP ratio elevation is not affected by the expression of ${ }^{\mathrm{mut}}$ SpiCee. Scale bars, $20 \mu \mathrm{m}$. (B, E, H, K, N) Data are mean \pm s.e.m. (C, F, I, L, O) Box-and-whisker plot elements: center line, mean; box limits, upper and lower quartiles; whiskers, s.d.; *** $P \leq 0.001$, (C, F, I, L) Mann-Whitney test, (O) KruskalWallis test. Exact $P$ values and number of replicates are provided in Table S1. 

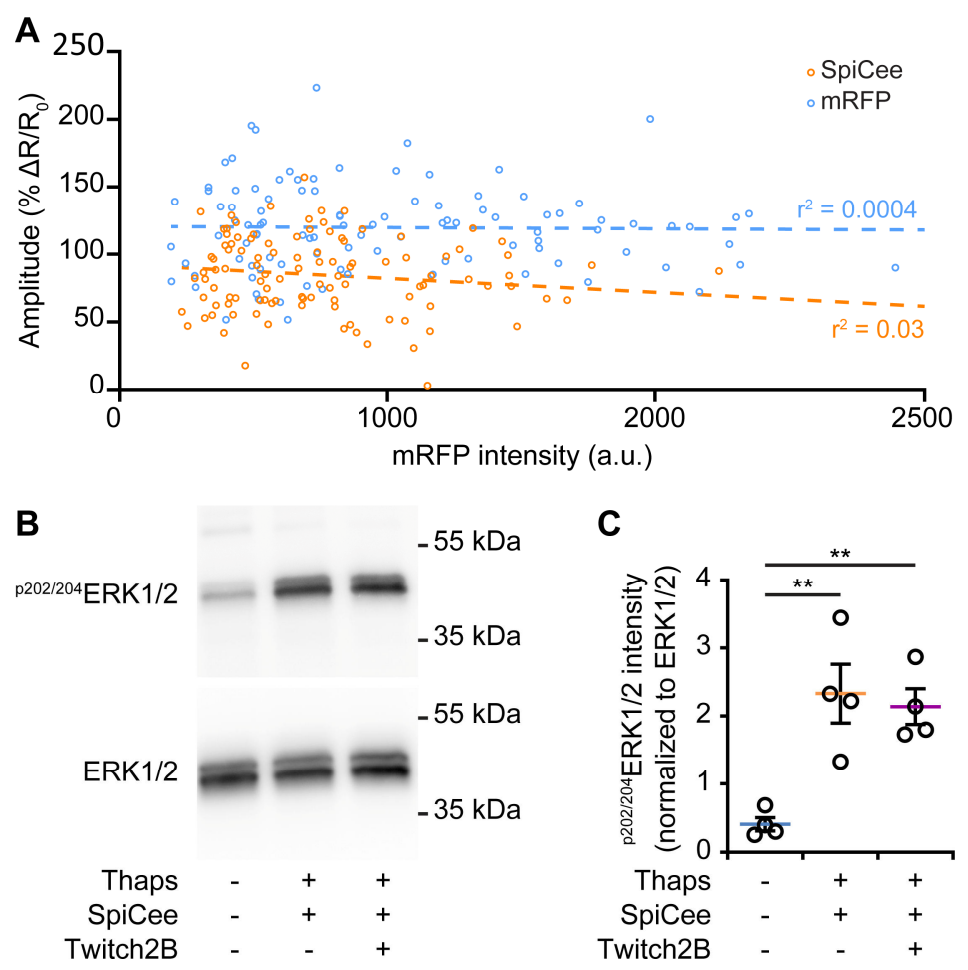

Figure S3. $\mathrm{Ca}^{2+}$ buffering by SpiCee is not affected by the level of expression or by co-expression of Twitch2B. Related to Figure 3. (A) The amplitude of the ATP-induced elevation in the FRET/CFP ratio was plotted against the mRFP fluorescence as a proxy of SpiCee or mRFP expression level. The dataset shown in Figure 3D-F was used. The level of mRFP fluorescence is correlated with the amplitude of the FRET/CFP ratio elevation neither in mRFP- (blue data) nor in SpiCee-expressing (orange data) cells. Data points were fitted to a straight line. In both cases the fit leads to close to horizontal line with a minimal $\mathrm{r}^{2}$ coefficient. (B) HEK293 cells expressing either SpiCee or SpiCee and Twitch2B were exposed to thapsigargin and the level of ERK1/2 phosphorylation was assessed. The calcium-induced elevation of ERK phosphorylation is not reduced in SpiCee and Twitch2B co-expressing cells compared to cells expressing SpiCee alone. (C) Data are mean \pm s.e.m. Individual data points are shown. ${ }^{*} P \leq 0.01$; ANOVA followed by Bonferroni post hoc tests. Exact $P$ values and number of replicates are provided in Table $\mathrm{S} 1$. 

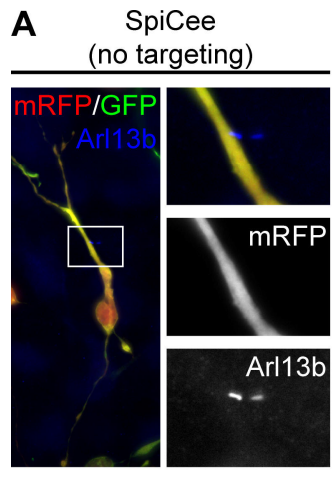

B
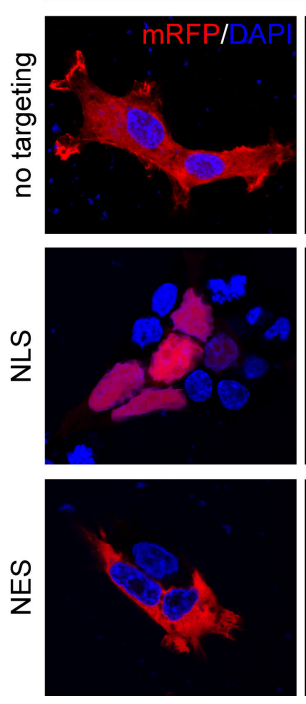

5HT6-SpiCee (primary cilium)
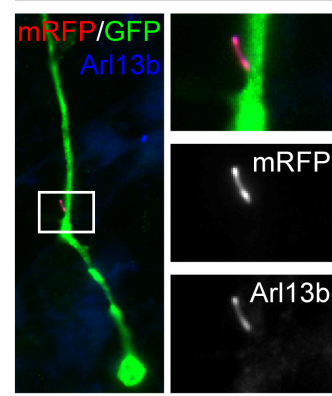

5HT6-hPMCA

(primary cilium)

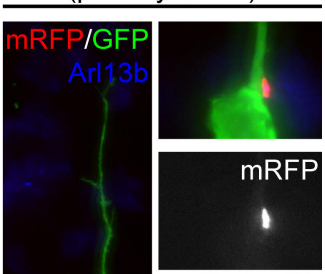

1

Arl13b

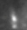

hPMCA
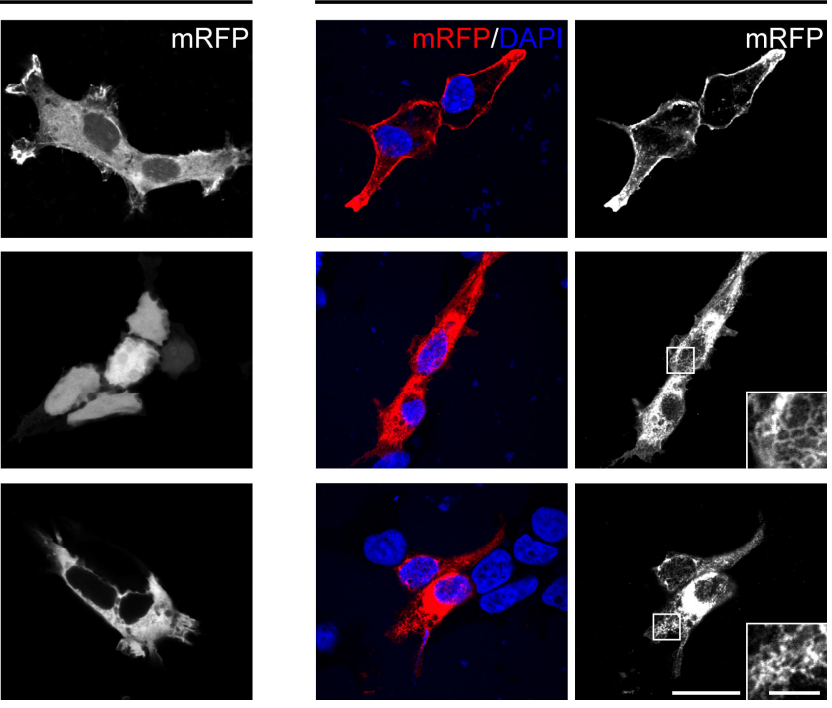

Figure S4. SpiCee can be targeted to membrane and intracellular comportments, in contrast to hPMCA. Related to Figure 6. (A) In cortical interneurons, SpiCee is found throughout the cytoplasm and excluded from the primary cilium stained with Arl13b (higher magnification in insets). When fused to the 5HT6 receptor, it is targeted to the primary cilium (middle). Like SpiCee, hPMCA can be targeted to this plasma membrane compartment using the 5HT6 receptor (right). (B) In HEK293 cells, SpiCee is found throughout the cytoplasm and to lesser extent in the nucleus whereas hPMCA expression is restricted to the plasma membrane. SpiCee expression can be restricted to the nucleus using a nuclear localization sequence (NLS) or to the cytoplasm using a nuclear export sequence (NES). In contrast, attempts to restrict or exclude hPMCA expression from the nucleus failed and led to its trapping to a compartment resembling the endoplasmic reticulum. Scale bars, $20 \mu \mathrm{m}$; in insets $5 \mu \mathrm{m}$. 
A

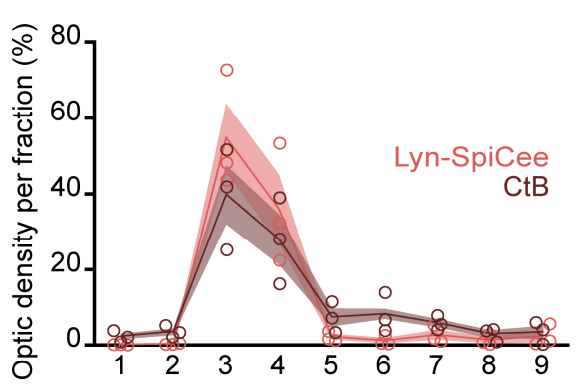

B

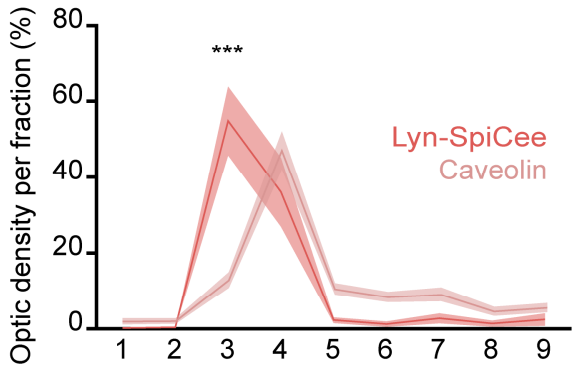

C

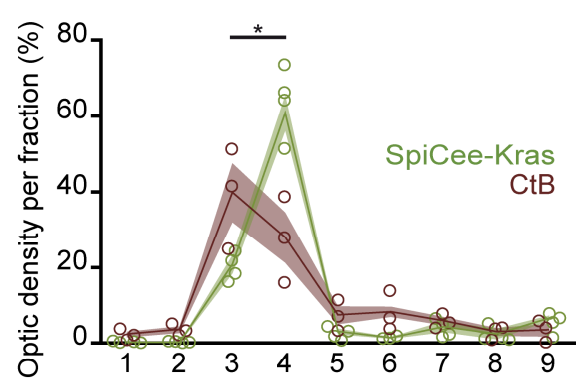

D

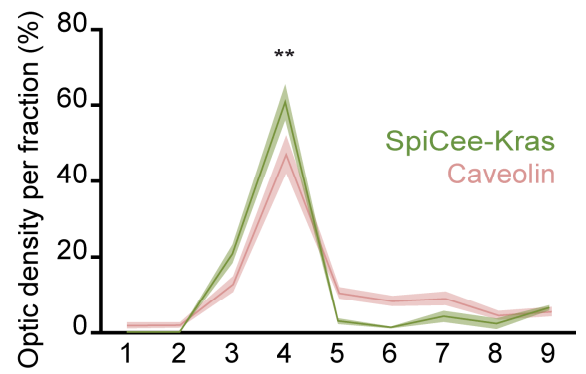

E

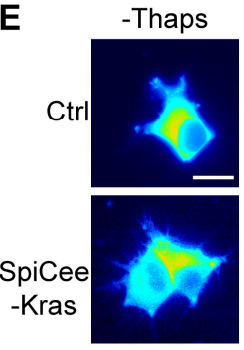

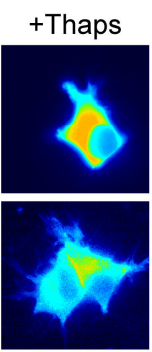

$\mathbf{F}$

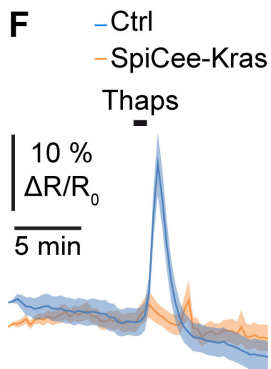

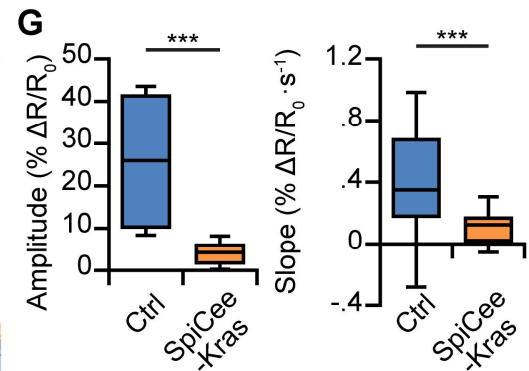

Figure S5. Biochemical characterization of SpiCee targeting. Related to Figure 6. (A, B) Lyn-SpiCee peaks in fractions 3 in a membrane fractionation assay. (A) It coincides with the expression profile of the $\beta$ subunit of cholera toxin ( $\mathrm{CtB}$, enriched in fractions 3 and 4, peaks in fraction 3) and (B) differs from the expression of Caveolin (enriched in fraction 4). SpiCee-Kras is mostly enriched in fraction 4, with a profile (C) distinct from $\mathrm{CtB}$, and (D) resembling Caveolin. (E) The Kras-targeted variant of Twitch2B was coexpressed with either mRFP (top row) or SpiCee-Kras (bottom row) in HEK293 cells. SpiCee-Kras reduces the thapsigargin-induced FRET channel fluorescence elevation of Twitch2B-Kras. (F) FRET/CFP ratio and (G) amplitude and slope quantifications from cells co-expressing either Twith2B-Kras and mRFP (blue trace and boxes) or Twith2B-Kras and SpiCee-Kras (orange trace and boxes). (A-D, F) Data are mean \pm s.e.m., (G) Box-and-whisker plot elements: center line, mean; box limits, upper and lower quartiles; whiskers, s.d.; $* P \leq 0.05$, *** $P \leq 0.01, * * * P \leq 0.001$, (A-D) Two-way ANOVA and Bonferroni post hoc tests, (G) Mann-Whitney test. Exact $P$ values and number of replicates are provided in Table S1. Data shown in Figure 6D is included here for additional comparisons. Individual data points are shown in Figure 6D when $\mathrm{n} \leq 10$. 

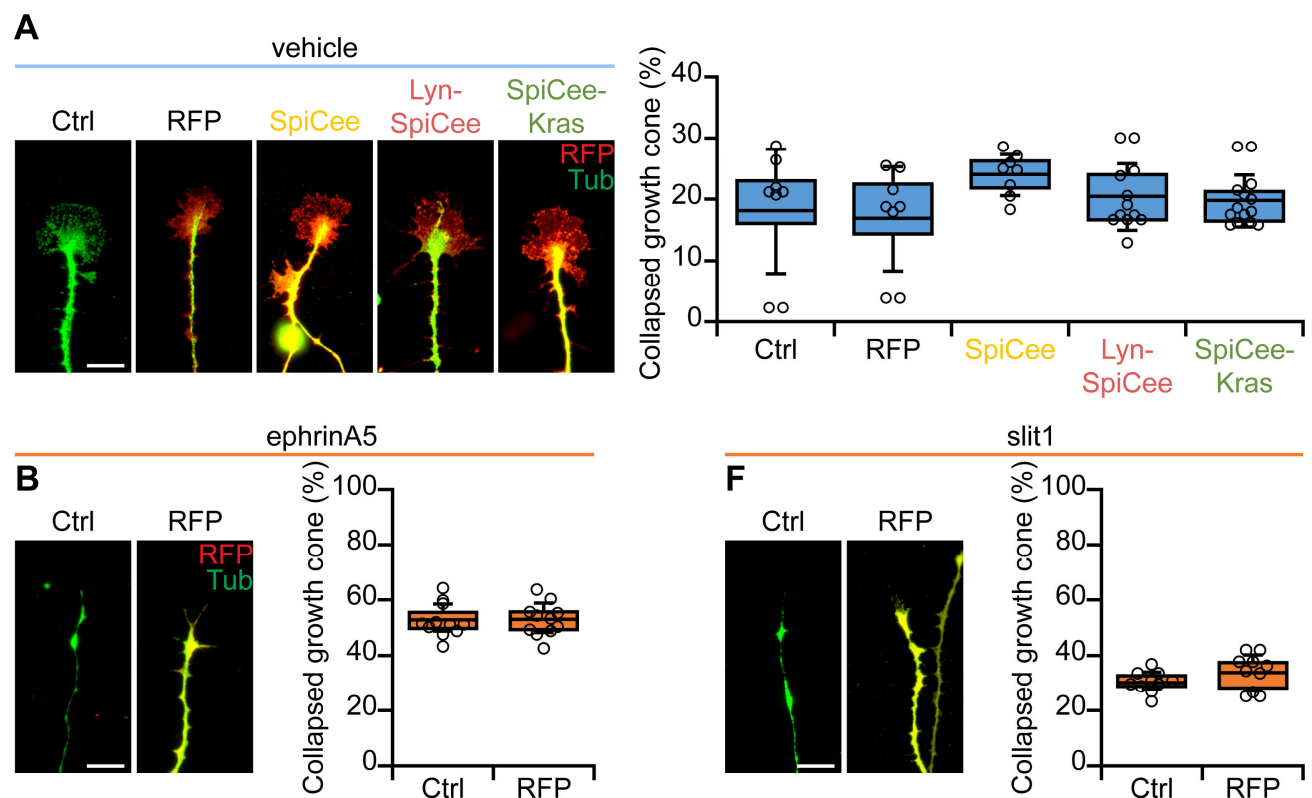

ephrinA5
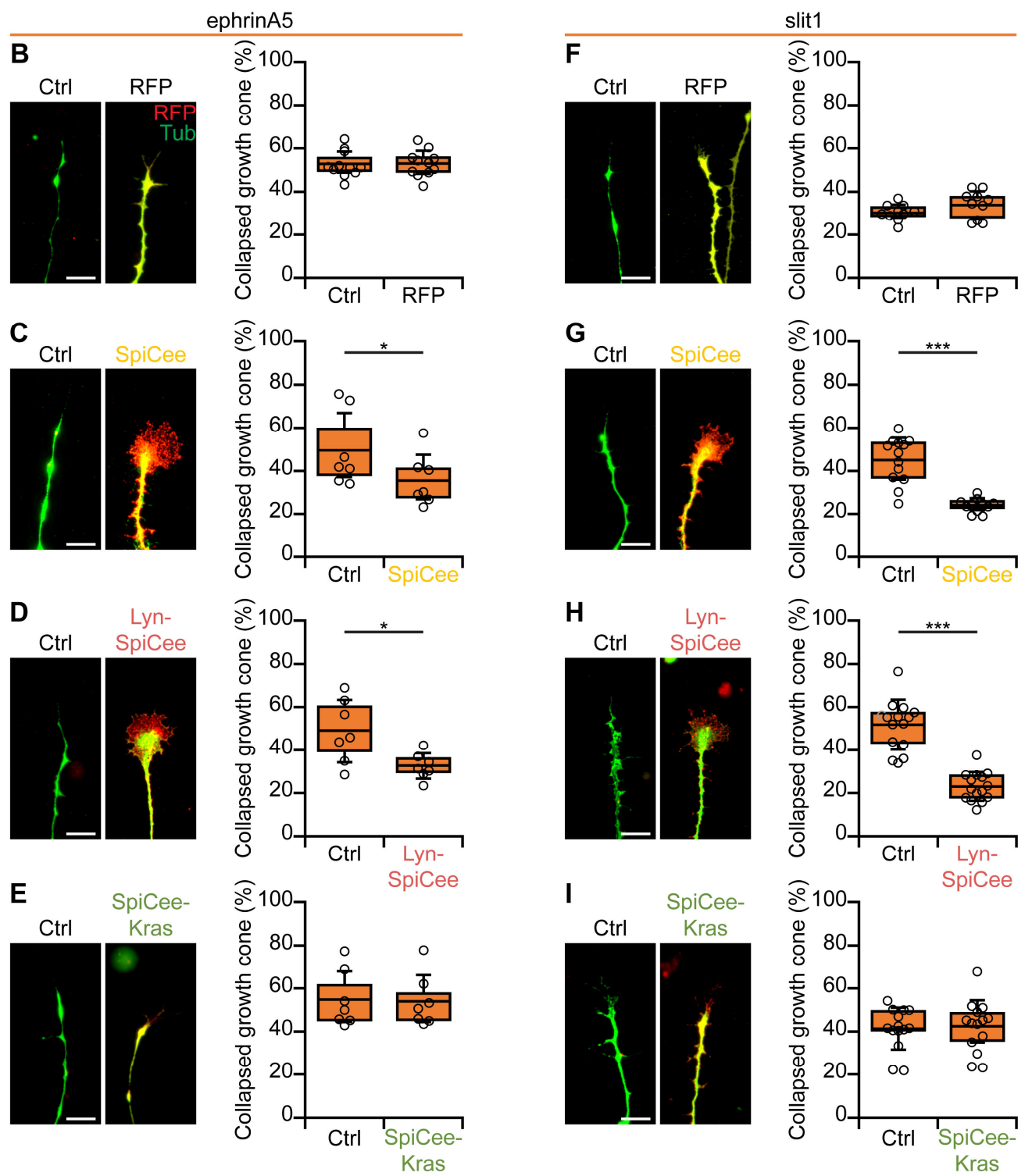

Figure S6. Cytosolic SpiCee and Lyn-SpiCee but not SpiCee-Kras prevent ephrinA5- and Slit1induced growth cone collapse. Related to Figure 6. Non-electroporated RGC axons display a fan-shaped growth cone at their tip which is not affected by the expression of mRFP, SpiCee, Lyn-SpiCee or SpiCeeKras. When exposed to (B) ephrinA5 or (F) slit1, mRFP-expressing growth cones exhibit the same collapse response than their non-electroporated neighbors of the same coverslips, (C, G) SpiCee- and (D, H) LynSpiCee-expressing axons are resistant to the repellent activity of ephrinA5 and slit1, that is observed in the non-electroporated axons from the same coverslip. (E, I) In contrast, SpiCee-Kras-expressing axons exhibit 
similar growth cone remodeling to their controls. Box-and-whisker plot elements: center line, mean; box limits, upper and lower quartiles; whiskers, s.d. Scale bar, $10 \mu \mathrm{m}$. (A) No significant differences are detected; Kruskal-Wallis test. (B-I) * $P \leq 0.05$; *** $P \leq 0.001$; Wilcoxon paired test. Exact $P$ values and number of replicates are provided in Table $\mathrm{S} 1$. A subset of the data shown in Figure $6 \mathrm{E}$ and $6 \mathrm{~F}$ is included here for additional comparisons with control growth cones from the same coverslips. 